\title{
Efficiency-House Optimization to Widen the Operation Range of the Double-Suction Centrifugal Pump
}

\author{
Wenjie Wang, ${ }^{1}$ Majeed Koranteng Osman, ${ }^{1,2}$ Ji Pei $\mathbb{D}^{1},{ }^{1}$ Shouqi Yuan, ${ }^{1}$ Jian Cao, \\ and Fareed Konadu Osman ${ }^{1}$ \\ ${ }^{1}$ National Research Center of Pumps, Jiangsu University, Zhenjiang 212013, China \\ ${ }^{2}$ Department of Mechanical Engineering, Wa Technical University, Wa, Ghana \\ ${ }^{3}$ Wuxi Dongfang Ship Research High Performance Boat Engineering Co., Ltd., Wuxi, China \\ Correspondence should be addressed to Ji Pei; jpei@ujs.edu.cn
}

Received 30 May 2020; Revised 16 August 2020; Accepted 8 September 2020; Published 26 September 2020

Academic Editor: Zong Woo Geem

Copyright ( 2020 Wenjie Wang et al. This is an open access article distributed under the Creative Commons Attribution License, which permits unrestricted use, distribution, and reproduction in any medium, provided the original work is properly cited.

Most pumping machineries have a problem of obtaining a higher efficiency over a wide range of operating conditions. To solve that problem, an optimization strategy has been designed to widen the high-efficiency range of the double-suction centrifugal pump at design $\left(Q_{\mathrm{d}}\right)$ and nondesign flow conditions. An orthogonal experimental scheme is therefore designed with the impeller hub and shroud angles as the decision variables. Then, the "efficiency-house" theory is introduced to convert the multiple objectives into a single optimization target. A two-layer feedforward artificial neural network (ANN) and the Kriging model were combine based on a hybrid approximate model and solved with swarm intelligence for global best parameters that would maximize the pump efficiency. The pump performance is predicted using three-dimensional Reynolds-averaged Navier-Stokes equations which is validated by the experimental test. With ANN, Kriging, and a hybrid approximate model, an optimization strategy is built to widen the high-efficiency range of the double-suction centrifugal pump at overload conditions by $1.63 \%, 1.95 \%$, and $4.94 \%$ for flow conditions $0.8 Q_{\mathrm{d}}, 1.0 Q_{\mathrm{d}}$, and $1.2 Q_{\mathrm{d}}$, respectively. A higher fitting accuracy is achieved for the hybrid approximation model compared with the single approximation model. A complete optimization platform based on efficiency-house and the hybrid approximation model is built to optimize the model double-suction centrifugal pump, and the results are satisfactory.

\section{Introduction}

There have been persistent efforts to reduce global fossil fuel consumption due to growing concerns of carbon emissions. This has made energy efficient centrifugal pumps a necessity since pumping power consumes about $10 \%$ of the global power share $[1,2]$. Centrifugal pumps are a group of turbomachinery with several applications ranging from domestic use to power plants and chemical and agricultural industries $[3,4]$. The double-suction centrifugal pump is a highly efficient pump as compared with the end suction pumps; however, like all centrifugal pumps, it has a common problem of efficiency reduction at nondesign flow conditions which translates into energy costs $[5,6]$. This requires an effective design optimization strategy to improve pump performance and save energy costs at the same time for the benefits of the manufacturer and the consumer.

The theory of pump design has over the years advanced from the one-dimensional Euler equation and empirical correction theory to a three-dimensional design theory such as direct design with numerical simulation. During this period, several optimization strategies have been applied by various researchers. Early design methods applied theoretical formulas to solve the mathematical expressions of pump performance for the design parameters. Though these were simple and straightforward, it was not so accurate. For example, theoretical models of net positive suction head (NPSH) and efficiency have been used for single-objective optimization in centrifugal and mixed-flow pumps $[7,8]$. The design of experiment (DOE) which is a mathematical 
approach has also been widely applied to optimize pump impellers for performance improvement within a shorter design period $[9,10]$.

Recently, there has been a shift from the single-objective optimization methods to the application of genetic algorithms (GAs) and surrogate models to find optimum global geometrical parameter combinations that can solve multiobjective optimization problems. Practically, the single-objective optimization cannot meet the design requirements for multiple objectives. Thus, compared with theoretical and design of experiment method, the surrogate model-based optimization methods are much more accurate and can control more design variables [11]. This has been applied to optimize the performance of a transonic airfoil [12]. Jin et al. [13] extensively reviewed the various surrogate models using their prediction accuracy, efficiency, and robustness and concluded that for higher-order nonlinear problems, neural networks should be used. The application of artificial neural networks has gained much recognition in turbomachinery optimization. In centrifugal pumps, the Radial bias neural network (RBNN) had the best prediction accuracy when compared with Kriging and the Response Surface Approximation surrogate models [14]. Also, Pei et al. [11] carried out a multiobjective optimization on the inlet pipe shape of a vertical inline pump using artificial neural network (ANN) and multiobjective genetic algorithm (MOGA) to increase the efficiency over a wide range. Furthermore, ANN was combined with particle swarm optimization (PSO) to establish that the multilayer neural network has a better prediction accuracy compared with the single-layer neural network in centrifugal pump efficiency optimization [2]. Meng used ANN and the nondominated sorting genetic algorithm (NSGA II) to perform a combination optimization to improve the reverse pump efficiency of an axial flow pump [15]. Other nature-inspired algorithms such as the artificial bee colony algorithm and PSO have been applied during performance optimization in centrifugal pumps $[16,17]$.

Whereas all these studies have focused on improving the pump performance at the nominal flow condition, the problem of obtaining a higher efficiency over a wide range of operating conditions has been rarely researched. To solve that problem, an optimization strategy has been designed to widen the efficiency range of the double-suction centrifugal pump over multiple working conditions. To improve the optimization accuracy and obtain the global optimal variables, an optimization method based on "efficiency-house" theory was developed to convert all the multiple objective functions into a single optimization objective. A hybrid approximate model was then proposed to improve the prediction accuracy. The fitting principles and accuracy test methods of a single approximation model and the hybrid approximation model were introduced and optimized at $0.8 Q_{\mathrm{d}}, 1.0 Q_{\mathrm{d}}$, and $1.2 Q_{\mathrm{d}}$ based on $A N N$ and Kriging models. This paper introduces an "efficiencyhouse" theory into a hybrid approximation model for optimization of the high-efficient range of the doublesuction centrifugal pump.

\section{Computational Research Domain}

2.1. Research Domain. The computational research domain is a 250GS40 double-suction centrifugal pump. The threedimensional model of the pump was built with Siemens NX. This is shown in Figure 1, and the design specifications of the pump are in Table 1. The flow domain is made up of the suction unit, a double-suction impeller with six twisted blades, and volute which serves as the pump outlet. The pump has a specific speed of 127 as defined in the following equation:

$$
n_{s}=\frac{3.65 n \sqrt{Q_{n}}}{60 H^{0.75}}
$$

2.2. Governing Equations. From the continuity equation, the time-dependent Reynolds-averaged Navier-Stokes (RANS) equation [18] was derived as

$$
\begin{aligned}
\frac{\partial \rho}{\partial t}+\frac{\partial}{\partial x_{j}}\left(\rho u_{j}\right) & =0, \\
\frac{\partial\left(\rho u_{i} u_{j}\right)}{\partial x_{j}}+\frac{\partial}{\partial t}\left(\rho u_{i}\right) & =\frac{\partial p}{\mathrm{~d} x_{i}}+\frac{\partial}{\mathrm{d} x_{j}}\left[\left(\mu+\mu_{t}\right)\left(\frac{\partial u_{i}}{\partial x_{j}}+\frac{\partial u_{j}}{\partial x_{i}}+\frac{2}{3} \frac{\partial u_{k}}{\partial x_{k}} \delta_{i j}\right)\right],
\end{aligned}
$$

where the ynamic viscosity and density are denoted by $\rho$ and $\mu$, respectively, and velocity is denoted by $u$, turbulent viscosity $\mu_{t}$, and $p$ pressure. The axis directions are represented by variables $i$ and $j$. Due to the combined advantages of both the $k-\square$ and $k-\varepsilon$ turbulence models having a good performance near the wall treatment $[19,20]$, the shear stress transport (SST $k-\square$ ) was chosen for turbulence closure to ensure accuracy during automatic wall treatment.

2.3. Test for Grid Sensitivity. A test of grid sensitivity was carried out to determine the total mesh elements suitable for the numerical simulation. This was to save computation time while maintaining accuracy of the simulation. First ANSYS ICEM was used to build structural hexahedral mesh for the whole computational domain. Further grid refinement was carried out and concentrated at the walls to attain higher precision and boundary motion features. This has been presented in Figure 2. Five independent grids were built and tested at the nominal flow condition. Since performance parameters like head and efficiency alone have been proved not to be sufficient enough for grid independence [21-23]; monitor points were mounted at the volute outlet (V1) and tongue (V2) to determine the grid sensitivity effect on the pressure distribution within the voulte. Figure 3 shows the arrangement of the monitoring points on the walls of the volute. The Grid Convergence Index (GCI) values were observed to be reducing with grid refinements. Though the discretization error is the smallest for grid number of 5847757 among the five different numbers of structured meshes, Mesh III was with GCI of $1.962 \%$ was adopted for as the final mesh for computation. This is because the effect of the mesh density on head, efficiency, and pressure 


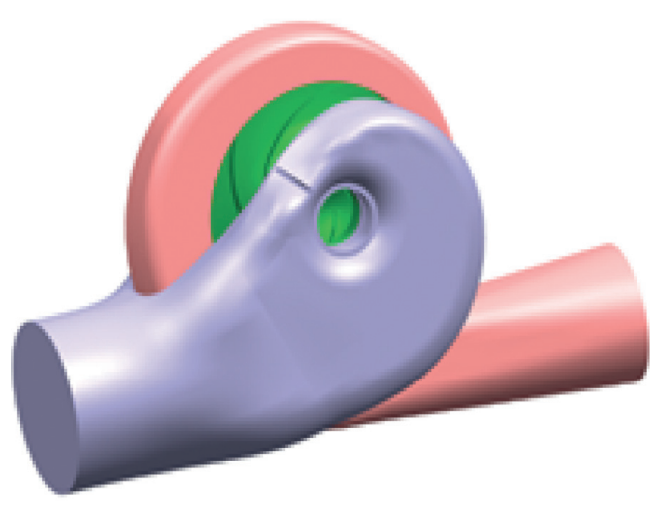

(a)

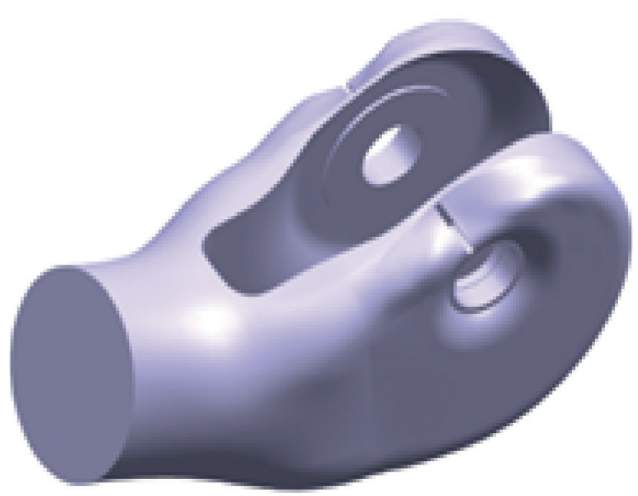

(b)

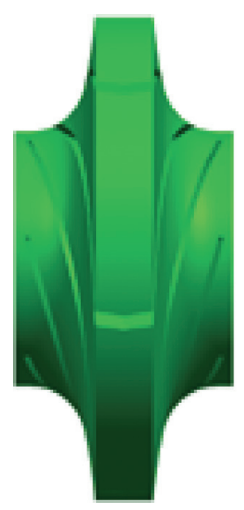

(c)

Figure 1: Computational model for model pump. (a) Complete model, (b) suction, and (c) impeller.

TABLE 1: Design specifications of model pump.

\begin{tabular}{lc}
\hline Design parameters & Value \\
\hline Flow rate, $Q_{\mathrm{d}}\left(\mathrm{m}^{3} / \mathrm{h}\right)$ & 500 \\
Head, $H(\mathrm{~m})$ & 40 \\
Rotational speed, $N(\mathrm{rpm})$ & 1480 \\
Suction diameter, $D_{\mathrm{s}}(\mathrm{mm})$ & 250 \\
Impeller inlet diameter, $D_{1}(\mathrm{~mm})$ & 192 \\
Impeller outlet diameter, $D_{2}(\mathrm{~mm})$ & 365 \\
Delivery diameter, $D_{\mathrm{d}}(\mathrm{mm})$ & 200 \\
Efficiency, $\eta$ & 84 \\
\hline
\end{tabular}

distribution of the pump was negligible when the total mesh elements reached 4,266,423 hexahedral control volumes. The statistics for grid convergence and sensitivity is shown in Table 2. For the final mesh, the average $Y+$ obtained was less than 50 and the distribution is presented in Figure 4.

2.4. Basic Calculation (CFX Pre) Setup. The ANSYS software package was used for the numerical investigations. The working fluid for the flow domain was water at $25^{\circ} \mathrm{C}$. The reference pressure was set to $0 \mathrm{~atm}$, and an isothermal heat transfer rate was chosen to put the system into thermal equilibrium. The pressure gradient is to be maintained accurately irrespective of the distance to the wall, and therefore the SST $(k-\omega)$ turbulence model was applied for automatic wall treatment due to additional effects in the viscous sublayer $[19,20]$. Smooth walls with a no-slip condition were assumed whereas the flow direction was set normal to the boundary condition. Referring to similar works, a turbulence intensity level of $5 \%$ was set $[6,24]$. To maintain accuracy and consistency during convergence, a high-resolution upwind scheme was set. This scheme locally adjusts the discretization to be as close to second order as possible, while ensuring the physical boundedness of the solution. The frozen-rotor condition was used as the frame change model. The advantage of this method is that it uses less computer resources than other frame change models, making it suitable for optimization studies. The domain interfaces were set to frozen rotor with a pitch angle of $360^{\circ}$ between the rotor and the stator. Conditions at the boundaries were pressure opening at inlet and flowrate at outlet. Iterations were set to a maximum of 700 , and convergence was achieved when the maximum residual values reached $10^{-5}$ and iterations were periodic stable. Performance indicators were calculated with reference to Gülich [25]. To calculate for the pump performance parameters, the results from the last 100 simulations were averaged.

2.5. Validation of Numerical Model. In order to validate the numerical model, experiments were conducted using the original pump model in an open test rig system. Figures 5 and 6 present the layout of the open test setup system that was used to perform the experiments. The highest point of the volute had an air bleed valve installed to get rid of entrained air within the system and also to supply cooling water to the mechanical seals. Pressure transducers with $0.5 \%$ measurement uncertainty were installed at the suction and discharge pipes. A flowmeter with an uncertainty of $0.07 \%$ was installed to regulate the flowrate from the pump discharge. To begin the tests, the pump was initially primed, paying much attention to the air bleed valve to get rid of any air in the pump. The motor was started until it reached its rated speed of $1480 \mathrm{rpm}$, and then the inlet and outlet pressures and the flow rate were recorded. Next, the outlet valve was systematically adjusted to the desired flow rate and the readings were taken. At each step, the motor speed was readjusted to maintain $1480 \mathrm{rpm}$. Hydraulic tests were performed at design and nondesign flow conditions. Afterwards, the discharge valve was gradually shut, and the power was cut to shut down the pump. The experimental process had a measurement uncertainty of $\pm 0.411 \%$, based on the Type B approach for evaluation of experimental uncertainty [26].

Since the experimentally measured total pump efficiency includes hydraulic efficiency, volumetric efficiency, and mechanical friction loss efficiency, the efficiency of numerical simulation is further processed. In the numerical simulation, the efficiency calculated is the hydraulic efficiency only. The experimental efficiency (overall efficiency) of the pump comprises the hydraulic efficiency $\eta_{h}$, the mechanical efficiency $\eta_{m}$, and the volumetric efficiency $\eta_{v}$; 


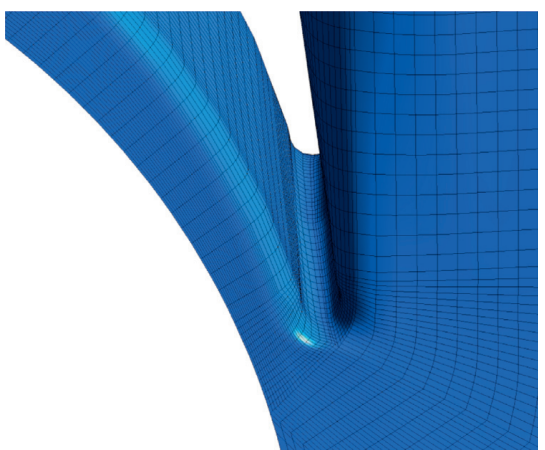

(a)

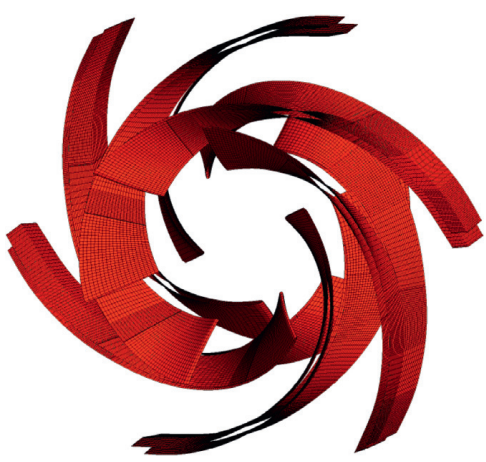

(b)

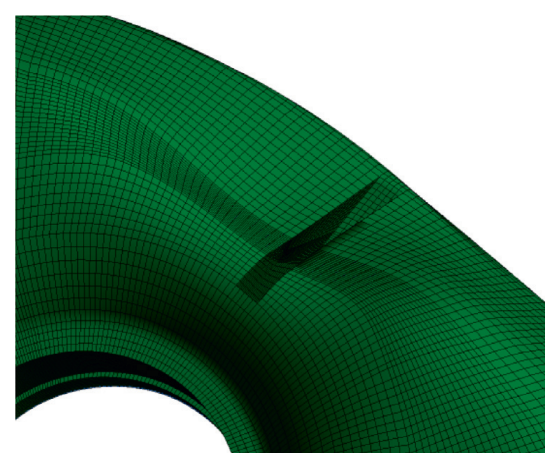

(c)

Figure 2: Mesh of calculation domain: (a) volute tongue, (b) impeller, and (c) suction tongue.

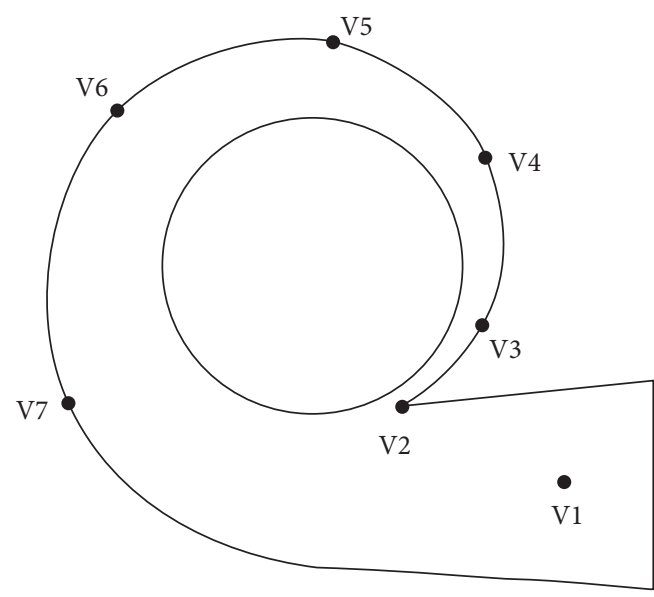

Figure 3: Arrangement of monitor points.

TABLE 2: Grid cells of the selected mesh.

\begin{tabular}{|c|c|c|c|c|c|c|}
\hline \multicolumn{2}{|l|}{ Item } & Mesh I & Mesh II & Mesh III & $\begin{array}{c}\text { Mesh } \\
\text { IV }\end{array}$ & Mesh V \\
\hline \multicolumn{2}{|c|}{ Total mesh } & 2878243 & 3679342 & 4266423 & 4958168 & 5847757 \\
\hline \multicolumn{2}{|c|}{ GCI (\%) } & 6.021 & 4.324 & 1.962 & 1.921 & 1.835 \\
\hline \multirow[t]{4}{*}{ Ratio } & $H / H_{1}$ & 1 & 1.156 & 1.221 & 1.220 & 1.221 \\
\hline & $\eta / \eta_{1}$ & 1 & & & & 1.304 \\
\hline & $p_{v 1} / p_{v 1,1}$ & 1 & 1.152 & 1.311 & 1.311 & 1.311 \\
\hline & $p_{v 2} / p_{v 2,1}$ & 1 & 1.156 & 1.282 & 1.282 & 1.282 \\
\hline
\end{tabular}

and therefore, in order to compare the numerical results with the test results, the numerical results has to be further scaled to match the test results. Thus, following the examples from $[24,27]$, the efficiency obtained from the simulation is further processed, before comparing with the test results. The mechanical efficiency is expressed as

$$
\eta_{m}=1-0.07 \frac{1}{\left(n_{s} / 100\right)^{(7 / 6)}}-0.02 .
$$

The volumetric efficiency is expressed as

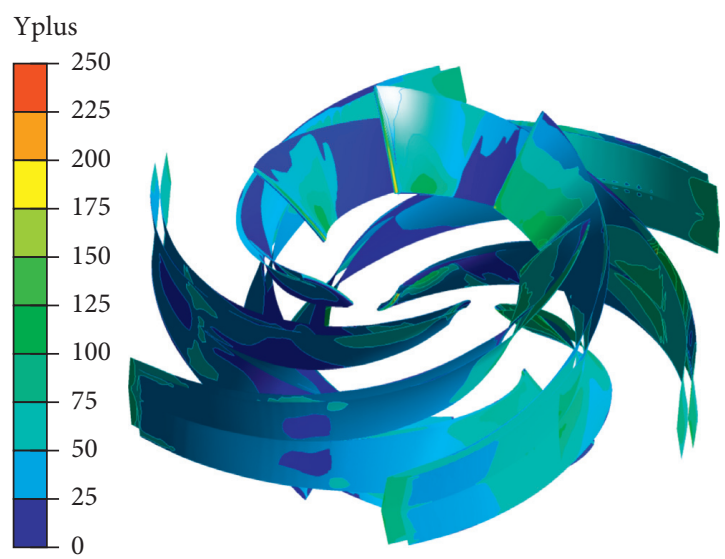

FIgURE 4: Blade surface $Y+$ distribution.

$$
\eta_{v}=\frac{1}{1+\left(0.68 n_{s}\right)^{(-2 / 3)}} .
$$

The relationship between the efficiencies is expressed as

$$
\eta=\eta_{h} \eta_{m} \eta_{v}
$$

This has been done in the previous studies [26]. Figure 7 compares the time-averaged transient simulation results at an impeller rotation of $3^{\circ}$ with the experimental test results. In the previous works on the same pump model [5], a time step dependency has been conducted to determine the influence of the time step on the transient simulation results. For the head curve, both test and numerical results follow the same trend for all flow conditions, with the maximum deviation not exceeding $1.2 \%$. For the efficiency curves, the maximum deviation occurs at an overload condition of $1.2 Q_{\mathrm{d}}$, representing about $2.49 \%$, whereas for part load conditions, the highest deviation between the test and numerical results was $1.67 \%$. In summary, the deviation between test and numerical values of both the head and efficiency for each flow condition are less than $3 \%$, which is within a reasonable range, and therefore the numerical simulation method adopted in this paper is reliable. 


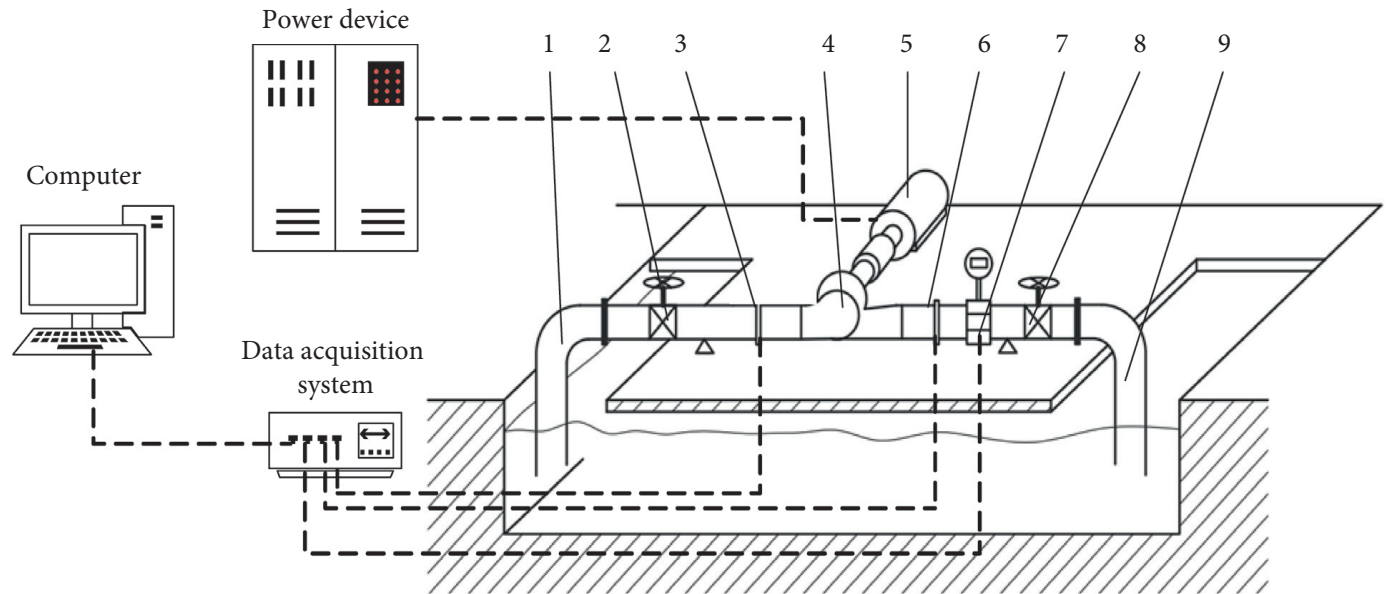

1: Inlet pipe

2(8): Valve

3(6): Pressure transducer

4: Tested pump
5: Driven motor

7: Magnetic flow meter

9: Outlet pipe

Figure 5: Schematics of the test setup.

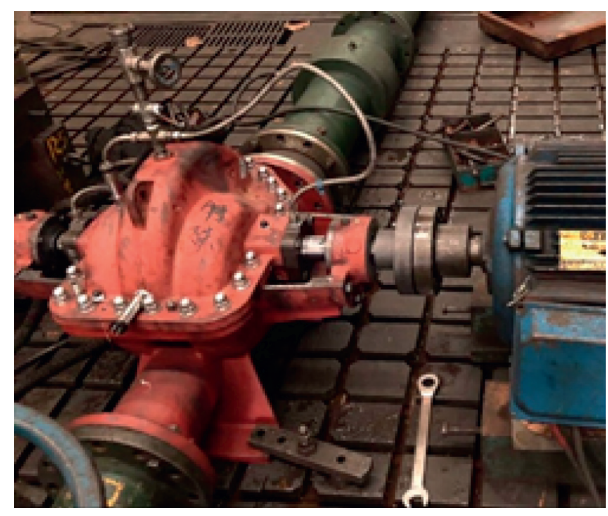

(a)

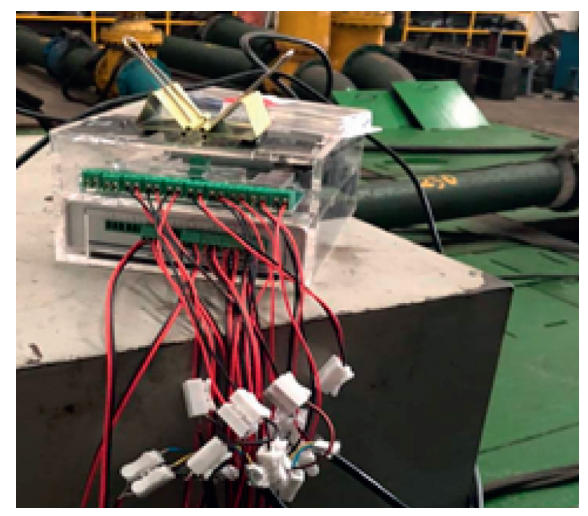

(b)

Figure 6: Test pump (a) and data acquisition device (b).

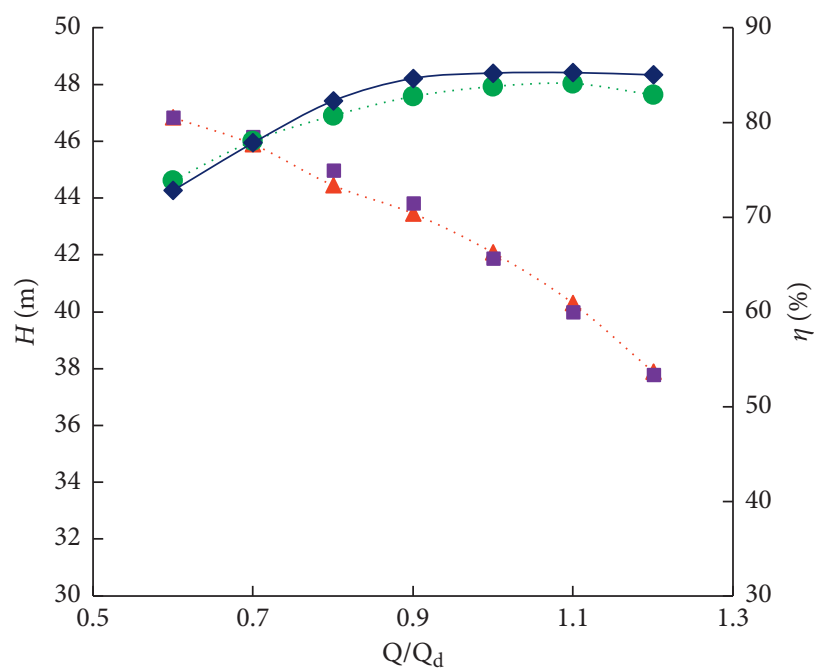
4. CFD - head
- CFD - efficiency
- TEST - head
$\checkmark$ TEST - efficiency

FIgURE 7: Validation with test results. 


\section{Optimization Strategy}

Figure 8 shows the flow chart of the strategy adopted for optimization. The initial process was to sample the decision variables based on the optimization objectives. Then, an orthogonal experimental test is carried out to obtain the effects of the pump geometry parameters on the optimization target. An "efficiency-house" theory is then applied to convert the optimization objectives of multiple operating conditions into a single objective, and a then a range analysis is then performed. This is to reduce the decision variables to improve upon the optimization speed. The final part is the optimization with a hybrid approximate model. The Latin hypercube test design method was used to randomly generate sample groups in the design space. The multiple optimization objectives are solved through numerical simulation and converted into a single objective based on the "efficiency-house" approach. A hybrid approximate model based on ANN and Kriging model was used to establish an approximate mathematical model for optimization. Thereafter PSO, a swarm intelligence algorithm was used to perform global optimization on approximate mathematical expressions to obtain the optimal design parameter combinations and the optimal optimization target of the double-suction pump impeller.

3.1. Objective Functions. For this study, the optimization objective was to maximize the pump efficiency for flow conditions $0.8 Q_{\mathrm{d}}, 1.0 Q_{\mathrm{d}}$, and $1.2 Q_{\mathrm{d}}$ concurrently. This was obtained by numerical model calculation. The optimization objective can be expressed as

$$
\max \left\{\begin{array}{l}
\eta_{0.8 Q_{\mathrm{d}}}=f_{1}, \\
\eta_{1.0 Q_{\mathrm{d}}}=f_{2}, \\
\eta_{1.2 Q_{\mathrm{d}}}=f_{3},
\end{array}\right.
$$

where $\eta_{0.8 Q_{\mathrm{d}}}=$ efficiency at $0.8 Q_{\mathrm{d}}, \eta_{1.0 Q_{\mathrm{d}}}=$ efficiency at design flow rate, and $\eta_{1.2 Q_{\mathrm{d}}}=$ efficiency at $1.2 Q_{\mathrm{d}}$.

The expression for calculating efficiency is

$$
\eta=\frac{\rho g H Q}{p_{\mathrm{s}}},
$$

where $\rho\left(\mathrm{kg} / \mathrm{m}^{3}\right)$ is the density, $H(\mathrm{~m})$ is the pump head, $Q$ $\left(\mathrm{m}^{3} / \mathrm{h}\right)$ is the flow discharge, and $P_{\mathrm{s}}(\mathrm{kW})$ the shaft power.

3.2. Decision Variables and Orthogonal Test. The structure of the double-suction impeller is compact and complex, making space constraints a limitation due the structure of the suction and volute casing. Therefore, the shape of the impeller was maintained by holding constant the inlet diameter $D_{1}$, the impeller outlet diameter $D_{2}$, the hub diameter $D_{\mathrm{h}}$, and the blade width at outlet $b_{2}$. The optimization therefore focused on the impeller hub and shroud angle effects on the blade profile, with all other parameters held constant. All eight geometry parameters were selected as decision variables for optimization. These are as follows: $\beta_{1 \_\mathrm{h}}$ and $\beta_{2 \_} \mathrm{h}$, the blade angles at hub inlet and exit, respectively; $\beta_{3 \_s}$ and $\beta_{4 \_s}$, the blade angle at the shroud inlet and exit; $\theta_{1 \_ \text {h }}$ and $\theta_{2 \_s}$, position of the leading edge at the hub and shroud; whereas $\Delta \theta_{1 \_ \text {h }}$ and $\Delta \theta_{2 \_s}$ are the leading edge wrap angle at the hub and shroud. The key to the parametric design of the impeller is to control of the blade profile. Figure 9 shows the axial projection of the impeller. The streamlines are made up of straight line and a circular arc $\mathrm{AB}$ for the hub and $\mathrm{CD}$ for the shroud. Points 3 and 1 are used to control the inclination angles of at the trailing edge. The angle and radius of the circular arc CD are controlled by point 4 and the angle and radius of arc $\mathrm{AB}$ are controlled by point 2 . The position of the leading edge at the hub and shroud EF is adjusted by the fourth-order Bézier curve. The control points can move freely.

The upper and lower limits for the decision variables were selected based on previous works [6]. The limits were then varied closely to create 4 parameter levels for each range of decision variable. Table 3 shows the range of decision variables and their respective levels. Based on this, the $L_{32}$ $\left(8^{4}\right)$ orthogonal scheme was designed for parametrization and simulated. Through numerical simulation, the orthogonal test results for the objective functions $\eta_{0.8 Q_{d}}, \eta_{1.0} Q_{d}$, and $\eta_{1.2 Q_{\mathrm{d}}}$ were obtained, as shown in Table 4 .

3.3. Range Analysis. It is necessary to conduct a range analysis to understand the extent to which each design variable affects the optimization target. To determine the variance $R$, the difference between the maximum and minimum values is analyzed for each factor at all the levels. The mathematical relation for the range analysis $[28,29]$ is expressed as

$$
\begin{aligned}
R & =\max \left(k_{1}, k_{2}, \ldots, k_{i}\right)-\min \left(k_{1}, k_{2}, \ldots, k_{i}\right), \\
k_{i} & =\frac{1}{N_{i}} \sum_{j=1}^{N_{i}} y_{i, j} \\
K_{i} & =\sum_{i=1}^{N_{i}} y_{i j}
\end{aligned}
$$

where $K_{i}$ is the sum of the values of the levels for each factor, $k_{i}$ is the average values, $y_{i, j}$ is the performance value for the factor, and $i$ and $j$ are the number of levels and number of factors, respectively.

Table 5 presents the level of influence of the geometrical parameters on the optimization objective for the three flow conditions. At $0.8 Q_{\mathrm{d}}, \beta_{2 \_\mathrm{h}}$ and $\beta_{4 \_s}$ had greater impact on efficiency. $\beta_{2 \_}$and $\beta_{1 \_}$h had the maximum influence on the optimization objective at the design point, whereas $\theta_{2 \_s}$ and $\Delta \theta_{2 \_ \text {s }}$ had the best influence at $1.2 Q_{\mathrm{d}}$. Hence, three best parameter combinations according to Table 3 were obtained as A3B1C3D1E4F4G3H2, A3B1C4D1E2F4G3H4, and A4B1C4D $3 \mathrm{E} 1 \mathrm{~F} 4 \mathrm{G} 3 \mathrm{H} 4$, for flow rates $0.8 Q_{\mathrm{d}}, 1.0 Q_{\mathrm{d}}$, and1.2Q $Q_{\mathrm{d}}$, respectively.

3.4. Efficiency-House Theory. From Table 5, it was found that the highest efficiency solution for each working condition could not be obtained at the same time. The "efficiencyhouse" theory was introduced to convert the efficiencies of the three working conditions into a single optimization target. The first step was to convert four operating condition points $\left(0.0 Q_{\mathrm{d}}, 0.8 Q_{\mathrm{d}}, 1.0 Q_{\mathrm{d}}\right.$, and $\left.1.2 Q_{\mathrm{d}}\right)$ to their flow 


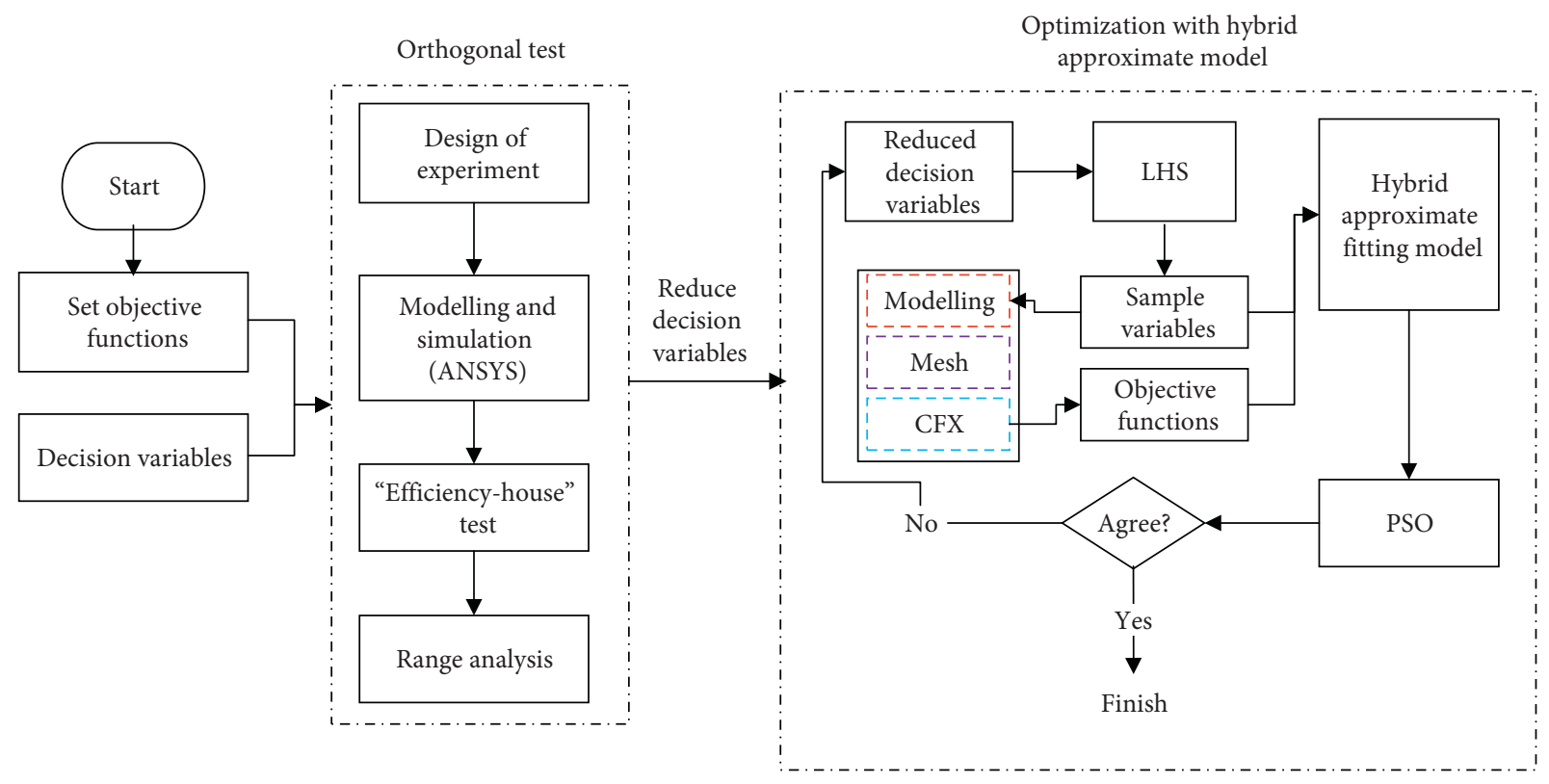

FIGURE 8: Flow chart of optimization strategy.

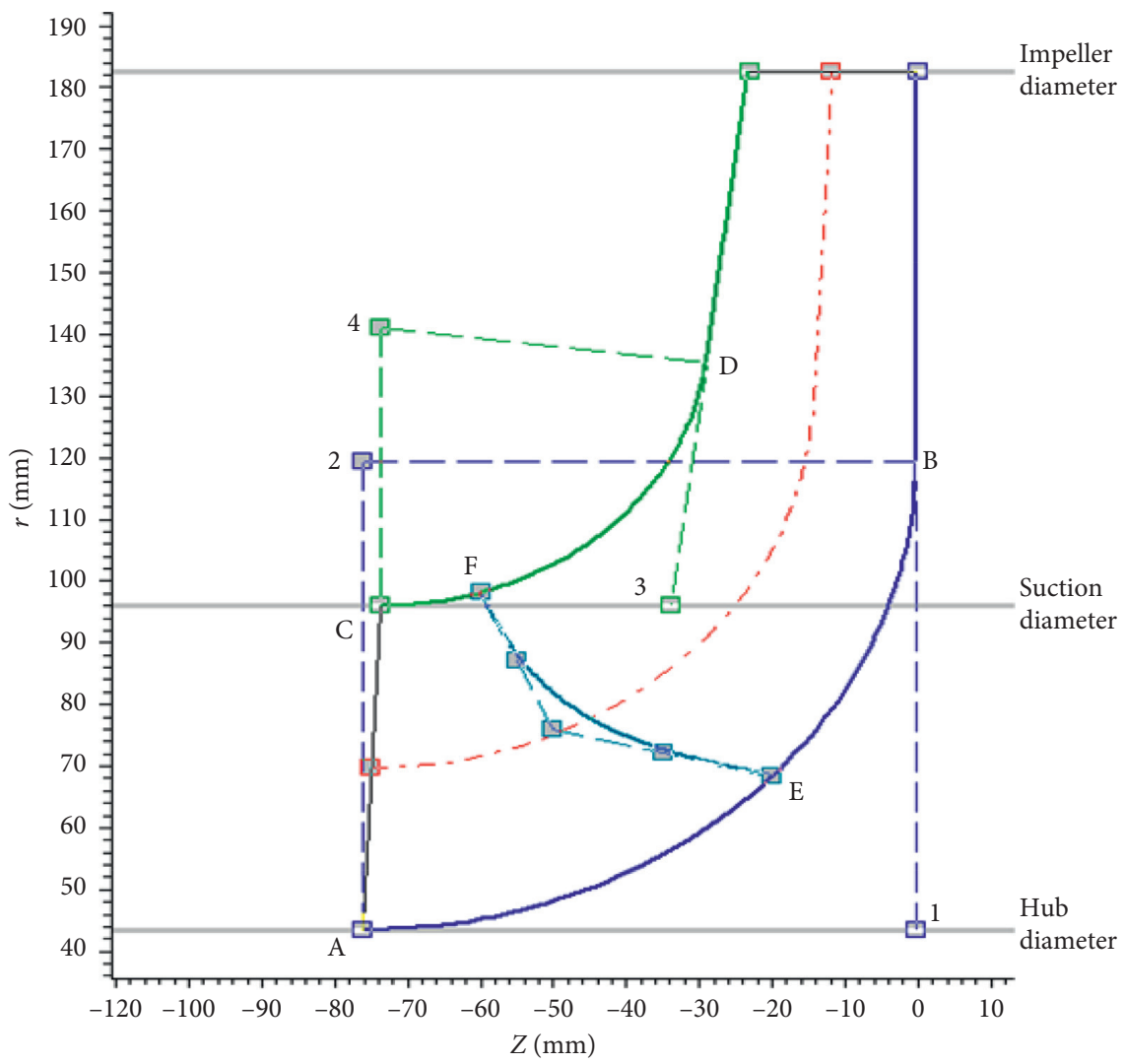

FIGURE 9: Impeller parameterization and meridional section.

coefficients. The corresponding efficiency was then used as a sample for the polynomial fitting in the following equation:

$$
\eta(\varphi)=a \varphi^{3}+b \varphi^{2}+c \varphi+d,
$$

where $\eta$ is the efficiency, $a, b, c$, and $d$ are cubic polynomial unknown coefficients, and $\varphi$ is a flow coefficient and is defined as 
TABLE 3: Range of decision variables.

\begin{tabular}{lcccccccc}
\hline \multirow{2}{*}{ Level } & $\mathrm{A}$ & $\mathrm{B}$ & $\mathrm{C}$ & $\mathrm{D}$ & $\mathrm{E}$ & $\mathrm{F}$ & $\mathrm{G}$ & $\mathrm{H}$ \\
& $\beta_{1 \_\mathrm{h}} /^{\circ}$ & $\beta_{2 \_\mathrm{h}}{ }^{\circ}$ & $\beta_{3 \_s}{ }^{\circ}$ & $\mathrm{B}_{4 \_s} /^{\circ}$ & $\theta_{1 \_\mathrm{h}} /^{\circ}$ & $\theta_{2 \_s} l^{\circ}$ & $\Delta \theta_{1 \_\mathrm{h}}{ }^{\circ}$ & $\Delta \theta_{2 \_s} l^{\circ}$ \\
\hline \multirow{2}{*}{ Original } & 17 & 29.43 & 15 & 29.43 & 0 & 0 & 143 & 143 \\
1 & 15 & 26 & 13 & 26 & -5 & -5 & 139 & 139 \\
2 & 17 & 28 & 15 & 28 & -2.5 & -2.5 & 143 & 143 \\
3 & 19 & 30 & 17 & 30 & 2.5 & 2.5 & 145 & 145 \\
4 & 21 & 32 & 19 & 32 & 5 & 5 & 148 & 148 \\
\hline
\end{tabular}

TABLE 4: Orthogonal test results.

\begin{tabular}{|c|c|c|c|}
\hline Level & $\eta_{0.8 Q_{d}}(\%)$ & $\eta_{1.0 Q_{\mathrm{d}}}(\%)$ & $\eta_{1.2 Q_{\mathrm{d}}}(\%)$ \\
\hline 1 & 84.70 & 88.03 & 86.87 \\
\hline 2 & 84.14 & 88.69 & 87.51 \\
\hline 3 & 84.86 & 89.21 & 87.60 \\
\hline 4 & 83.31 & 88.16 & 87.59 \\
\hline 5 & 82.25 & 87.41 & 86.45 \\
\hline 6 & 83.86 & 87.26 & 84.61 \\
\hline 7 & 83.17 & 87.20 & 86.04 \\
\hline 8 & 84.58 & 88.43 & 87.25 \\
\hline 9 & 83.72 & 87.88 & 87.43 \\
\hline 10 & 83.68 & 87.83 & 86.70 \\
\hline 11 & 85.07 & 88.44 & 85.61 \\
\hline 12 & 83.99 & 88.70 & 87.80 \\
\hline 13 & 84.25 & 88.62 & 88.35 \\
\hline 14 & 84.87 & 87.68 & 85.07 \\
\hline 15 & 83.74 & 88.02 & 87.40 \\
\hline 16 & 85.00 & 88.77 & 86.77 \\
\hline 17 & 84.96 & 88.66 & 86.28 \\
\hline 18 & 83.79 & 87.51 & 85.31 \\
\hline 19 & 83.99 & 87.88 & 85.27 \\
\hline 20 & 83.92 & 87.85 & 85.66 \\
\hline 21 & 84.70 & 89.40 & 88.82 \\
\hline 22 & 84.44 & 88.98 & 86.74 \\
\hline 23 & 83.29 & 87.83 & 87.37 \\
\hline 24 & 83.91 & 88.52 & 87.54 \\
\hline 25 & 83.85 & 88.36 & 86.94 \\
\hline 26 & 84.17 & 88.90 & 87.88 \\
\hline 27 & 84.40 & 88.93 & 87.99 \\
\hline 28 & 83.77 & 87.79 & 85.69 \\
\hline 29 & 84.15 & 87.87 & 87.72 \\
\hline 30 & 83.86 & 88.43 & 85.41 \\
\hline 31 & 84.89 & 88.18 & 87.09 \\
\hline 32 & 83.01 & 87.17 & 84.72 \\
\hline
\end{tabular}

$$
\varphi=\frac{Q}{\omega D_{2}^{3}} .
$$

Figure 10 is a sketch map of the efficiency-house model. The grey area under the curve, $S$, is the "efficiency-house," which is a single indicator for evaluating whether the efficiency interval is widened or not. Using a definite integration, the area under the quadratic curve is obtained by integrating the following equation:

$$
S=\int_{0}^{\varphi_{l}} \eta \mathrm{d} \varphi
$$

The three optimization models (model A, model B, and model C) obtained from the 32 sets of orthogonal experiments and range analysis were modelled according to the above steps, and the results are shown in Table 6. The area $S$ of the efficiency-house in Model $\mathrm{A}$ is the largest among the 35 sets of data. Table 6 is reanalyzed by range analysis to obtain the effect of the geometry parameters on the single optimization objective in Figure 11 to determine the level of influence of the decision variables on the area $S$ of the efficiency-house. In summary, the decision variables $\beta_{2} \mathrm{~h}$ $(\mathrm{B}), \beta_{4 \_s}(\mathrm{D}), \theta_{1 \_\mathrm{h}}(\mathrm{E})$, and $\theta_{2 \_s}(\mathrm{~F})$ had the most influence on the single optimization target based on efficiency-house. Therefore, there four geometry parameters were set as decision variables for the optimization with the hybrid approximate model.

3.5. Latin Hypercube Sampling (LHS). The orthogonal experiment design only optimizes each parameter under the condition of four levels, so a more accurate optimization method is required. However, from the results of the range analysis for the single optimization objective, the decision variables were reduced to four geometry parameters of the double-suction impeller, namely, $\beta_{2 \_}, \beta_{4 \_s}, \theta_{1 \_\mathrm{h}}$, and $\theta_{2 \_s}$. Therefore, based on the reduced decision variables, the design variable range has been redefined in Table 7. Taking the efficiency-house area $S$ as optimization target, the efficiencies under the three operating conditions should be greater than the efficiency of the original pump, and the head is lower than the original pump head by no more than $5 \%$. The mathematical model to determine the optimal design of the double-suction pump under multiple operating conditions is

$$
\begin{cases}\max & S, \\ \text { s.t. } & \eta_{0.8} \geq 84.9, \eta_{1.0} \geq 88.0, \eta_{1.2} \geq 85.0, \\ \text { s.t. } & H_{0.8} \geq 39.7, H_{1.0} \geq 38.5, H_{1.2} \geq 34.6 .\end{cases}
$$

The Latin hypercube test design method was used to randomly generate design points for the construction of the surrogate model. This is a method of approximately random sampling from a multivariate parameter distribution and belongs to the stratified sampling technique [15]. Forty design schemes were generated using the Latin hypercube method, as shown in Table 8. Each of the design schemes was simulated for the efficiency under $0.8 Q_{\mathrm{d}}, 1.0 Q_{\mathrm{d}}$, and $1.2 Q_{\mathrm{d}}$ flow conditions as shown in Table 9. The efficiency-house theory was used to then convert the three objectives into a single target for each of the design schemes in Table 10. It can be clearly seen that the $S$ value of these 40 sets of design schemes were mostly higher than the $S$ values of the optimal model A (Table 6) which indicates that the upper and lower limits of the design parameters are reasonable and a better solution can be found in this range.

3.6. Optimization with Approximate Model. The approximate model theory is mainly used for complex engineering problems that are difficult to describe with mathematical expressions and is generally called "black box problem." At present, the most widely used approximate models in engineering design are artificial neural network (ANN), 
TABLE 5: Range analysis.

\begin{tabular}{cccccccccc}
\hline & & & $\mathrm{B}$ & $\mathrm{C}$ & $\mathrm{D}$ & $\mathrm{E}$ & $\mathrm{F}$ & $\mathrm{G}$ & $\mathrm{H}$ \\
\hline \multirow{3}{*}{$R(\%)$} & $0.8 \mathrm{Qd}$ & 0.435 & 1.031 & 0.198 & 0.784 & 0.358 & 0.467 & 0.436 & 0.334 \\
& $1.0 \mathrm{Qd}$ & 0.753 & 0.832 & 0.444 & 0.550 & 0.227 & 0.409 & 0.227 & 0.509 \\
& $1.2 \mathrm{Qd}$ & 0.959 & 0.936 & 0.862 & 1.018 & 0.547 & 1.126 & 0.616 & 1.575 \\
\hline
\end{tabular}

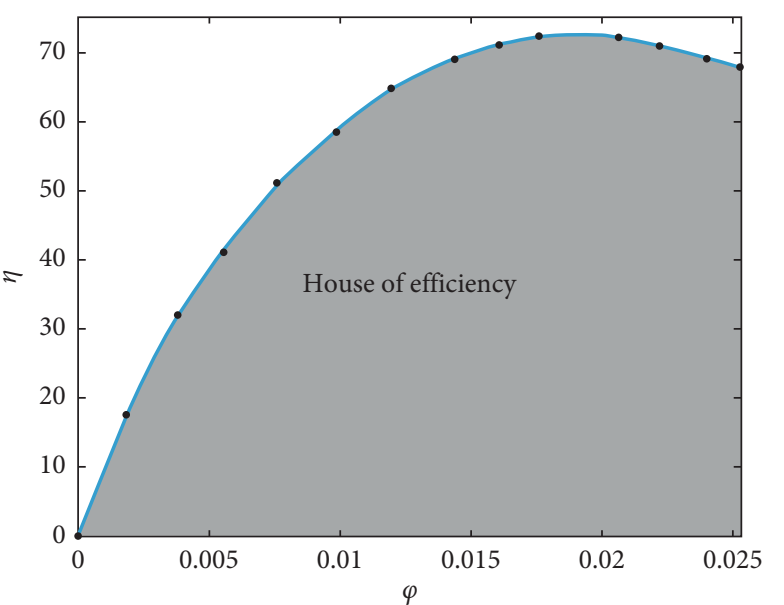

- Evs. q

- Polyfit

Figure 10: Sketch map of "efficiency-house" model.

Kriging, polynomial response surface (RSM) models. Jin [13] stated that, for higher-order nonlinear problems, neural networks have strong nonlinearity, whereas Kriging has higher accuracy. Therefore, two single approximate models, ANN and Kriging, were considered for the optimization process.

3.6.1. Artificial Neural Network. For this study, a two-layer feedforward artificial neural network with sigmoid hidden neurons and linear output neurons was adopted. The Levenberg-Marquardt algorithm was adopted since it is the fastest of all traditional or improved networks [6]. The tanh activation function was used because it has much better recognition accuracy for multilayer neural networks. The mathematical relation for the ANN function is written as equation (13), the activation function, tanh (14), and the linear function as equation (15):

$$
\begin{aligned}
y & =g\left(\sum_{j=1}^{n} w_{j}^{2} \times f\left(\sum_{k=1}^{m} w_{k}^{1}{ }_{j} x_{k}+b_{n}^{1}\right)\right)+b^{2}, \\
f(x) & =\left[\frac{2}{\left(1+e^{-2 x}\right)}\right]-1, \\
g(x) & =a x+b
\end{aligned}
$$

where $w$ is the weight coefficient, $b$ is the threshold, and superscripts 1 and 2 represent the coefficients from the first layer to the second layer and from the hidden layer to the output layer respectively.
TABLE 6: "Efficiency-house" of orthogonal tests.

\begin{tabular}{lc}
\hline Model & $S$ \\
\hline 1 & 9.0108 \\
2 & 8.8285 \\
3 & 8.8981 \\
4 & 8.7494 \\
5 & 8.5743 \\
6 & 8.8071 \\
7 & 8.7726 \\
8 & 8.9455 \\
9 & 8.8705 \\
10 & 8.8197 \\
11 & 8.9320 \\
12 & 8.8157 \\
13 & 8.9214 \\
14 & 8.9795 \\
15 & 8.8492 \\
16 & 8.9430 \\
17 & 8.9189 \\
18 & 8.7994 \\
19 & 8.7767 \\
20 & 8.7942 \\
21 & 8.9178 \\
22 & 8.7892 \\
23 & 8.7850 \\
24 & 8.8110 \\
25 & 8.7835 \\
26 & 8.8254 \\
27 & 8.8760 \\
28 & 8.7750 \\
29 & 8.9819 \\
30 & 8.6676 \\
31 & 9.0407 \\
B & 8.6526 \\
C & 9.0885 \\
\hline & 8.9772 \\
& 8.9057 \\
\hline &
\end{tabular}

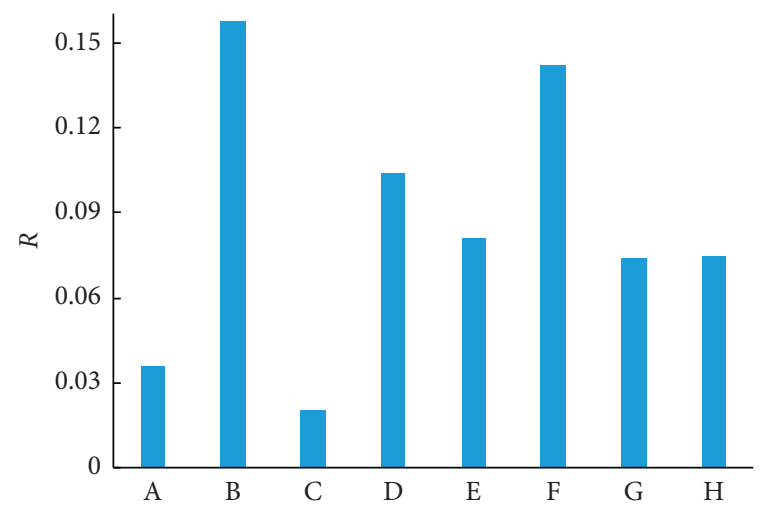

FIGURE 11: Level of influence of decision variable on S. 
TABLE 7: LHS range of design parameters.

\begin{tabular}{lcccc}
\hline Input & $\beta_{2 \_\mathrm{h}}$ & $\beta_{4 \_\mathrm{s}}$ & $\theta_{1 \_\mathrm{h}}$ & $\theta_{2 \_s}$ \\
\hline Lower bound & 26424 & 26524 & 266145 & 267145 \\
Upper bounds & 26930 & 27030 & 271155 & 272155 \\
\hline
\end{tabular}

TABle 8: Cases designed by LHS.

\begin{tabular}{lcccc}
\hline Level & $\beta_{2 \_\mathrm{h}}$ & $\beta_{4 \_ \text {s }}$ & $\theta_{1 \_\mathrm{h}}$ & $\theta_{2 \_\mathrm{s}}$ \\
\hline 1 & 26.8 & 25.7 & 146.0 & 147.1 \\
2 & 29.3 & 27.9 & 149.7 & 152.3 \\
3 & 26.4 & 29.2 & 148.7 & 153.4 \\
4 & 27.5 & 29.3 & 148.8 & 152.2 \\
5 & 29.6 & 28.4 & 146.4 & 148.9 \\
$\ldots$ & $\ldots$ & $\ldots$ & $\ldots$ & $\ldots$ \\
16 & 25.7 & 29.7 & 153.9 & 146.1 \\
17 & 29.0 & 26.6 & 153.1 & 151.1 \\
18 & 25.0 & 27.7 & 154.6 & 152.9 \\
19 & 26.0 & 28.8 & 146.8 & 149.6 \\
20 & 26.1 & 27.1 & 150.6 & 151.3 \\
21 & 25.1 & 25.9 & 149.9 & 152.5 \\
22 & 29.5 & 29.9 & 147.3 & 146.3 \\
23 & 28.5 & 24.4 & 151.9 & 150.9 \\
24 & 28.7 & 26.1 & 152.3 & 145.7 \\
25 & 28.0 & 29.7 & 147.6 & 150.1 \\
$\ldots$ & $\ldots$. & $\ldots$. & $\ldots$ & $\ldots$ \\
36 & 28.2 & 28.2 & 150.8 & 147.9 \\
37 & 26.5 & 24.1 & 149.5 & 148.7 \\
38 & 28.9 & 26.9 & 152.9 & 149.1 \\
39 & 27.4 & 24.3 & 151.3 & 148.4 \\
40 & 27.0 & 28.7 & 153.6 & 151.9 \\
\hline
\end{tabular}

3.6.2. Kriging Model. The Kriging model is widely used for interpolation of nonlinear problems, and a series of methods suitable for various situations can be derived. For example, Ordinary Kriging is a univariate local linear optimal unbiased estimation method, while Ordinary Co-Kriging can be extended to two or more variables. Its mathematical expression is written as

$$
\begin{aligned}
Y & =\beta F(s)+z(s), \\
\operatorname{Cov}\left[z\left(s_{i}\right), z\left(s_{j}\right)\right] & =\sigma^{2} r\left(s_{i}, s_{j}\right) .
\end{aligned}
$$

In equation (9), $\mathbf{S}=\left(\begin{array}{lllll}s_{1} & s_{2} & \ldots & s_{m}\end{array}\right)^{T}$ is the $m$-dimensional design variable, and $\mathbf{Y}=\left(\begin{array}{llll}y_{1} & y_{2} & \ldots & y_{m}\end{array}\right)^{T}$ is the $m$-dimensional response value $[30,31], F(s)$ is the global regression model, $\beta$ is the regression coefficient, and $z(s)$ is the correlation function, subject to the local deviation created by the regression model based on the mean value of zero and the variance is not zero, generally Gaussian function equation (17), and $z\left(s_{i}\right)$ is the covariance between any two and where $r\left(s_{i}, s_{j}\right)=$ $\prod_{d=1}^{q}-\theta^{d}\left|s_{i}^{d}-s_{j}^{d}\right|^{p^{d}}$.

3.6.3. Particle Swarm Optimization (PSO). PSO is a widely used swarm intelligence algorithms because it has excellent
TABle 9: Efficiency under three conditions.

\begin{tabular}{lccc}
\hline Level & $\eta_{0.8 Q_{\mathrm{d}}}(\%)$ & $\eta_{1.0 Q_{\mathrm{d}}}(\%)$ & $\eta_{1.2 \mathrm{Q}_{\mathrm{d}}}(\%)$ \\
\hline 1 & 85.67 & 89.28 & 88.61 \\
2 & 85.30 & 88.80 & 88.83 \\
3 & 85.61 & 89.17 & 88.53 \\
4 & 85.22 & 89.04 & 88.68 \\
5 & 84.92 & 88.54 & 88.25 \\
$\ldots$ & $\ldots$ & $\ldots$ & $\ldots$ \\
$\ldots$ & $\ldots$ & $\ldots$ & $\ldots$ \\
15 & 85.73 & 89.42 & 88.69 \\
16 & 84.89 & 89.00 & 88.46 \\
17 & 85.55 & 88.88 & 88.65 \\
18 & 86.03 & 89.15 & 86.35 \\
19 & 85.72 & 89.34 & 88.97 \\
20 & 85.87 & 89.29 & 88.79 \\
21 & 86.21 & 89.80 & 89.16 \\
22 & 84.60 & 88.23 & 88.10 \\
23 & 85.65 & 89.34 & 88.99 \\
24 & 84.98 & 89.21 & 88.77 \\
25 & 85.18 & 88.88 & 88.52 \\
$\ldots$ & $\ldots$ & $\ldots$ & $\ldots$ \\
$\ldots$ & $\ldots$ & $\ldots$ & 88.53 \\
35 & 85.45 & 89.04 & 88.40 \\
36 & 84.82 & 88.88 & 89.17 \\
37 & 86.26 & 89.65 & 88.57 \\
38 & 85.05 & 88.89 & 88.11 \\
39 & 84.38 & 88.45 & \\
40 & 85.61 & 88.88 &
\end{tabular}

global search capabilities. Its origin is from the process of birds searching for food using acoustics of echolocation. The algorithm imitates the cluster behavior and sets a series of behavior rules for each individual, so that the group can complete complex tasks. The basic mathematical model of particle swarm optimization is

$$
\begin{aligned}
& v_{i, t+1}=v_{i, t}+c_{1} r_{1 i}\left(p_{\text {best }}-s_{i, t}\right)+c_{2} r_{2 i}\left(g_{\text {best }}-s_{i, t}\right), \\
& s_{i, t+1}=s_{i, t}+v_{i, t+1},
\end{aligned}
$$

where $s_{i, t}$ and $v_{i, t}$ represent the position and velocity; $c_{1}$ and $c_{2}$ are the velocity coefficients of learning from the particle's own optimal position and the velocity component learned from the group's optimal position, and $r_{1 i}$ and $r_{2 i}$ are the random numbers, respectively, the value ranges from 0 to 1 $[32,33]$.

3.7. Hybrid Approximate Model. This paper combines ANN and the Kriging model and uses the approximate model to fit the correlation coefficient $R^{2}$ to solve the mixed model coefficients. The specific construction process is shown in equations (13) and (14). The swarm intelligence algorithmparticle swarm optimization is the used to perform global optimization on approximate mathematical expressions to obtain the optimal design parameters combination and the optimal optimization target of the double-suction pump 
TABLe 10: Efficiency-house of design.

\begin{tabular}{|c|c|}
\hline Level & $S$ \\
\hline 1 & 9.1254 \\
\hline 2 & 9.1436 \\
\hline 3 & 9.1257 \\
\hline 4 & 9.0765 \\
\hline 5 & 9.0675 \\
\hline 6 & 9.1459 \\
\hline 7 & 9.1593 \\
\hline 8 & 9.2032 \\
\hline 9 & 9.1163 \\
\hline 10 & 9.1646 \\
\hline 11 & 9.0951 \\
\hline 12 & 9.0851 \\
\hline 13 & 9.1125 \\
\hline 14 & 8.9159 \\
\hline 15 & 9.1201 \\
\hline 16 & 8.9991 \\
\hline 17 & 9.1699 \\
\hline 18 & 9.0651 \\
\hline 19 & 9.1509 \\
\hline 20 & 9.1779 \\
\hline 21 & 9.1895 \\
\hline 22 & 9.0421 \\
\hline 23 & 9.1377 \\
\hline 24 & 9.0044 \\
\hline 25 & 9.0837 \\
\hline 26 & 9.1818 \\
\hline 27 & 9.0551 \\
\hline 28 & 9.1167 \\
\hline 29 & 9.0867 \\
\hline 30 & 9.1925 \\
\hline 31 & 9.147 \\
\hline 32 & 9.0587 \\
\hline 33 & 9.0867 \\
\hline 34 & 8.962 \\
\hline 35 & 9.114 \\
\hline 36 & 9.0004 \\
\hline 37 & 9.2256 \\
\hline 38 & 9.0585 \\
\hline 39 & 8.9602 \\
\hline 40 & 9.1713 \\
\hline
\end{tabular}

impeller. Figure 12 shows the hybrid approximate model fitting procedure:

$$
\begin{aligned}
& y_{\mathrm{en}}(X)=\omega_{1} y_{1}(X)+\omega_{2} y_{2}(X), \\
& \left\{\begin{array}{l}
\text { Find: } \omega_{1}, \\
\text { Max: } R^{2}=1-\frac{\sum_{i=1}^{m}\left(y_{\mathrm{en}}-\widehat{y_{\mathrm{en}}}\right)^{2}}{\sum_{i=1}^{m}\left(y_{\mathrm{en}}-\overline{y_{\mathrm{en}}}\right)^{2}} \\
\omega_{1}+\omega_{2}=1, \quad 0 \leq \omega_{1} \leq 1,
\end{array}\right.
\end{aligned}
$$

where $y_{1}(X)$ is the ANN model, $y_{2}(X)$ is the Kriging model, $R^{2}$ is the correlation coefficient, $m$ is the number of samples, $y_{\text {en }}$ is the actual response value, $\widehat{y_{\mathrm{en}}}$ is the approximate model response value, and $\overline{y_{\text {en }}}$ represents the mean value of the approximate model response [34, 35].
The 40 groups of data generated by the above LHS were divided into two groups: $70 \%$ of the samples (28 groups) were used to train artificial neural networks and Kriging and $30 \%$ of the samples (12 groups) were used to verify the accuracy of the fit. After the approximate model was successfully established, the accuracy of the approximate model was evaluated using the $R$-square error analysis method. From Figure 13, the $R^{2}$ values of ANN was 0.8 , whereas Kriging fitting accuracy had $R^{2}$ value is about 0.88 . However, both $R^{2}$ values were less than 0.9 . For the composite model, the fitting accuracy of the hybrid approximate model reached 0.95167, as shown in Figure 14, which proved that the ANN/Kriging hybrid approximate model has a higher fitting accuracy than each single approximate model.

\section{Discussion of Results}

4.1. Validation of Optimization Results. The ANN, Kriging, and hybrid approximation models established were used for optimization calculations. After convergence, the optimal design parameter combination and the optimal $S$ results are shown in Table 11. It can be seen from the table that the optimized efficiency area $S$ of the three approximate models is higher than the maximum value in the 40 groups of samples in Table 10. In order to verify the accuracy of the optimization results, the three sets of design parameters obtained were subjected to three-dimensional modeling and numerical simulation, and the true $S$ value of each group of solutions is calculated and compared with the predicted values in Table 12. Although the optimization results obtained by ANN are the most ideal, the errors between the predicted values and the true values are large, indicating that the fitting accuracy of ANN and Kriging approximate models was lower than that of the composite approximate model which had a deviation of $0.003 \%$. This proved the optimization method reliable for further analysis.

4.2. Comparison of External Characteristics. The numerical simulation results from hybrid approximate optimization model for each flow condition are compared with those of the original model in Table 13. Comparing the efficiencies, the optimized efficiency has been increased by $1.63 \%, 1.95 \%$, and $4.94 \%$ under flow conditions $0.8 Q_{\mathrm{d}}, 1.0 Q_{\mathrm{d}}$, and $1.2 Q_{\mathrm{d}}$, respectively. Although the head was slightly reduced under each working condition, the reduction in head lies within the optimization objective of no more than $5 \%$.

In order to study the flow loss inside different components, the head distribution analysis was performed on the original model and the optimized model. The head distribution in different flow channels before and after optimization is shown in Table 14. For each flow condition, the impeller function was weakened by the optimization, thereby reducing the input power significantly. This explains the corresponding increase in efficiency for the three working conditions after optimization. In addition, although the impeller's workability was reduced after optimization, the hydraulic loss inside the suction chamber and the volute 


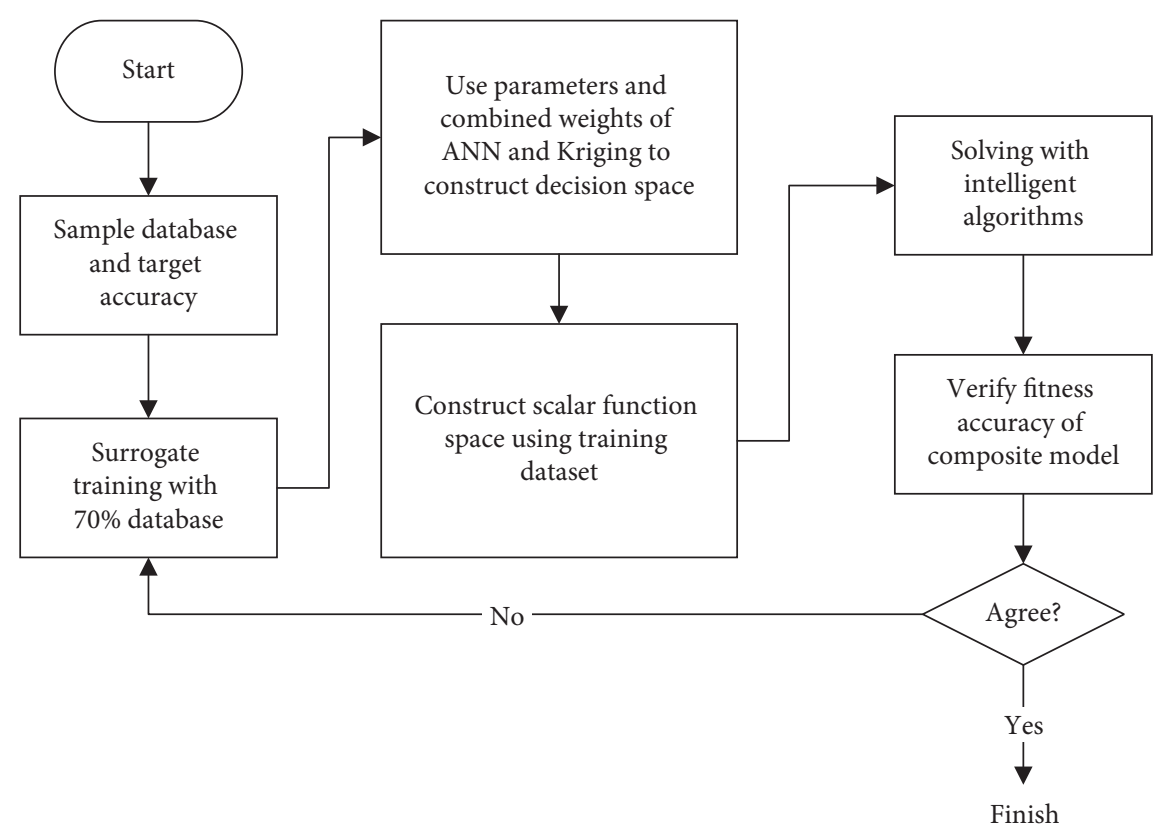

FIGURE 12: Fitting process of hybrid approximate model.

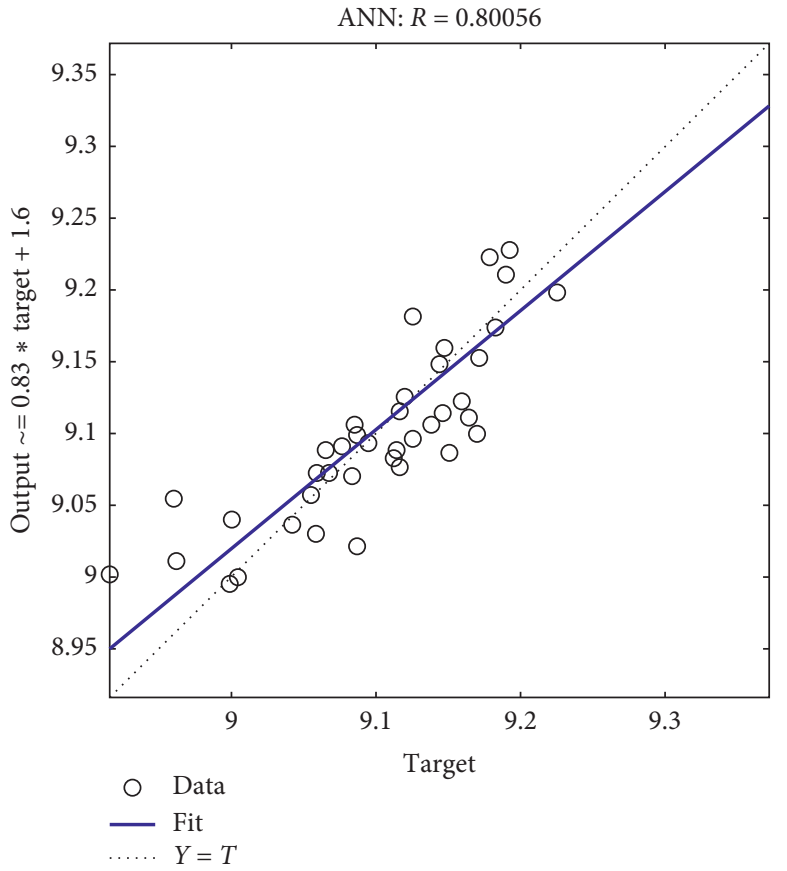

(a)

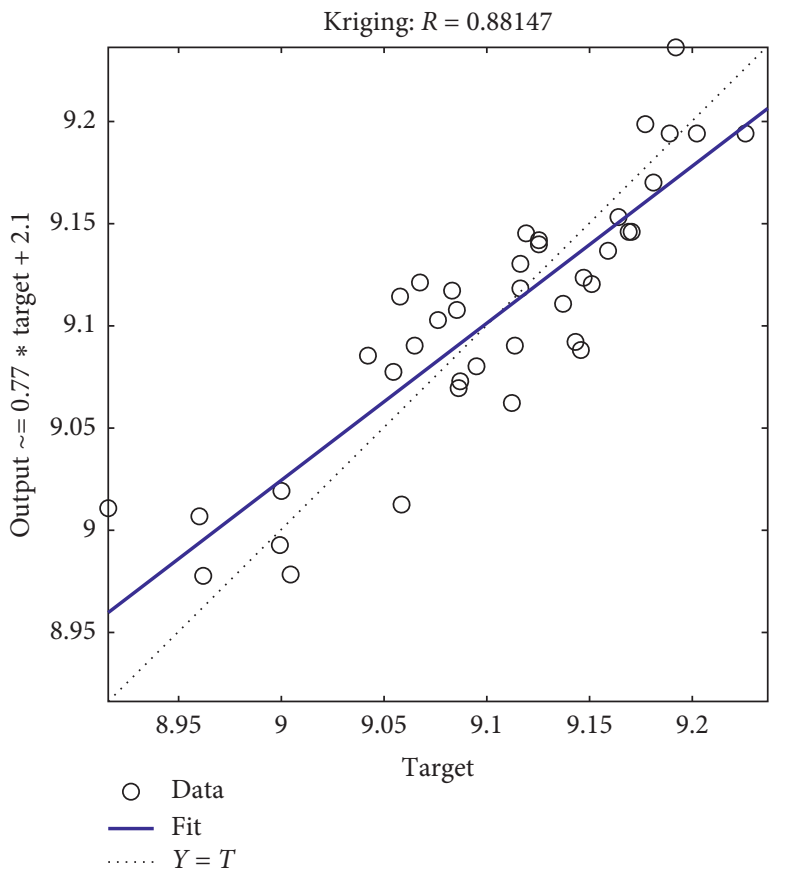

(b)

FIGURE 13: $R$-square analysis.

has been reduced to varying degrees, so the total head still meets the design requirements.

To clearly see and compare the changes in the highefficiency zone before and after optimization, the efficiency curve for optimal model A in the orthogonal test and the efficiency from the hybrid approximation is compared with that of the original model in Figure 15.
From the results, the high-efficiency zone of the doublesuction centrifugal pump was widened, especially under overload conditions, which solves the problem of the steep drop in efficiency of the original pump model at overload conditions. At part loads, there was a marginal improvement in the efficiency of the composite approximation model as compared with the optimization 


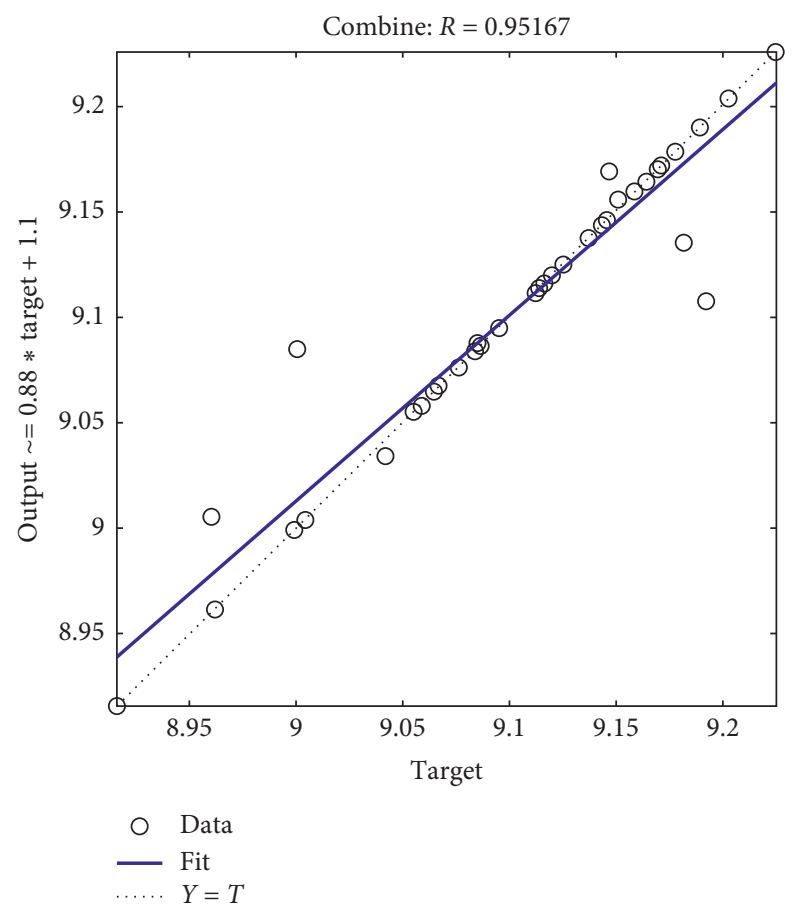

FIgURE 14: Fitting accuracy of composite model.

TABLE 11: Optimization results of different surrogate model.

\begin{tabular}{lccccc}
\hline & $\beta_{2 \_\mathrm{h}}$ & $\beta_{4 \_\mathrm{s}}$ & $\theta_{1 \_\mathrm{h}}$ & $\theta_{2 \_\mathrm{s}}$ & $S$ \\
\hline ANN & 25.0235 & 28.8943 & 150.5623 & 152.8956 & 9.2268 \\
Kriging & 24.8156 & 29.2201 & 149.7032 & 154.2331 & 9.2257 \\
Hybrid & 24.8707 & 29.2325 & 148.8955 & 154.0112 & 9.2263 \\
\hline
\end{tabular}

TABLe 12: Error analysis of optimization results.

\begin{tabular}{lccc}
\hline & $S$ (predict) & $S($ CFD) & Error (\%) \\
\hline ANN & 9.2268 & 9.0243 & 2.24 \\
Kriging & 9.2257 & 9.1296 & 1.05 \\
Hybrid & 9.2263 & 9.2260 & 0.003 \\
\hline
\end{tabular}

TABLE 13: Comparison of performance.

\begin{tabular}{|c|c|c|c|c|c|c|}
\hline & \multicolumn{2}{|c|}{$\eta(\%)$} & \multirow{2}{*}{ Deviation (\%) } & \multicolumn{2}{|c|}{ Head (m) } & \multirow{2}{*}{ Deviation (\%) } \\
\hline & Original & Optimized & & Original & Optimized & \\
\hline $0.8 Q_{\mathrm{d}}$ & 84.92 & 86.3 & 1.63 & 41.84 & 41.65 & 0.45 \\
\hline $1.0 Q_{\mathrm{d}}$ & 87.99 & 89.71 & 1.95 & 40.53 & 39.32 & 2.99 \\
\hline $1.2 Q_{\mathrm{d}}$ & 85.00 & 89.2 & 4.94 & 36.51 & 36.18 & 0.90 \\
\hline
\end{tabular}

TABLE 14: Hydraulic head distribution of optimized and original cases.

\begin{tabular}{lccccc}
\hline Flowrate & Model & Suction $(\mathrm{m})$ & Impeller $(\mathrm{m})$ & Volute $(\mathrm{m})$ & Power $(\mathrm{kw})$ \\
\hline \multirow{2}{*}{$0.8 \mathrm{Q}_{\mathrm{d}}$} & Original & -1.307 & 48.620 & -4.342 & 53.291 \\
& Optimized & -0.258 & 45.920 & -3.725 & 52.957 \\
\hline \multirow{2}{*}{$1.0 \mathrm{Q}_{\mathrm{d}}$} & Original & -0.167 & 44.196 & -3.141 & 62.735 \\
& Optimized & -0.150 & 42.372 & -2.673 & 59.877 \\
\hline \multirow{2}{*}{$1.2 \mathrm{Q}_{\mathrm{d}}$} & Original & -0.200 & 41.243 & -4.187 & 70.206 \\
& Optimized & -0.210 & 39.196 & -2.582 & 66.554 \\
\hline
\end{tabular}




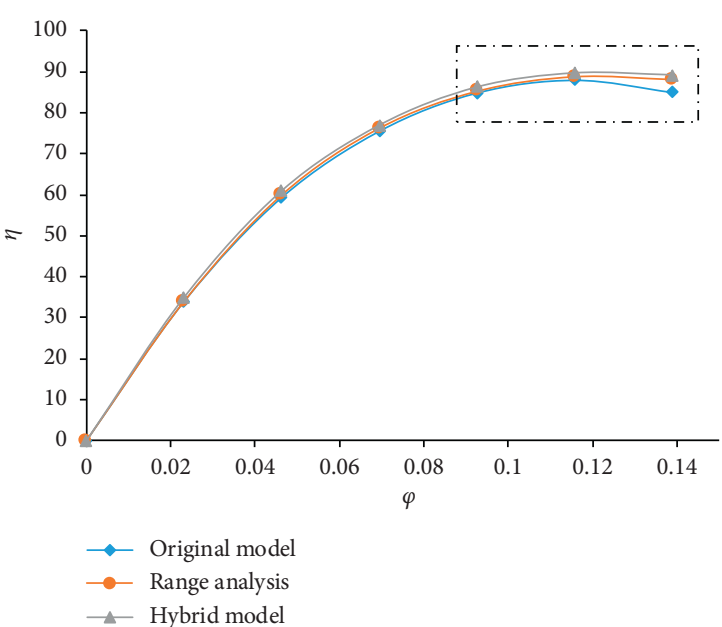

(a)

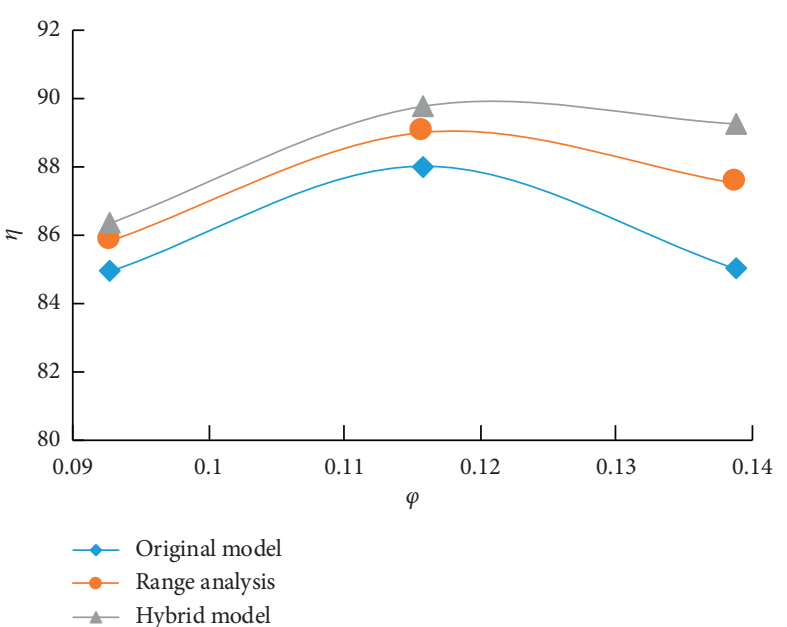

(b)

FIgURE 15: Comparison of high-efficiency zone before and after optimization.
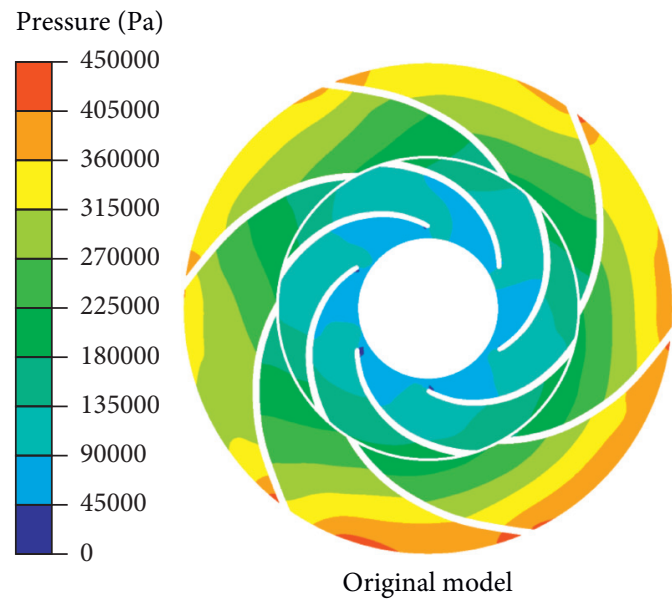

Figure 16: Comparison of pressure distribution at $0.8 Q_{\mathrm{d}}$.

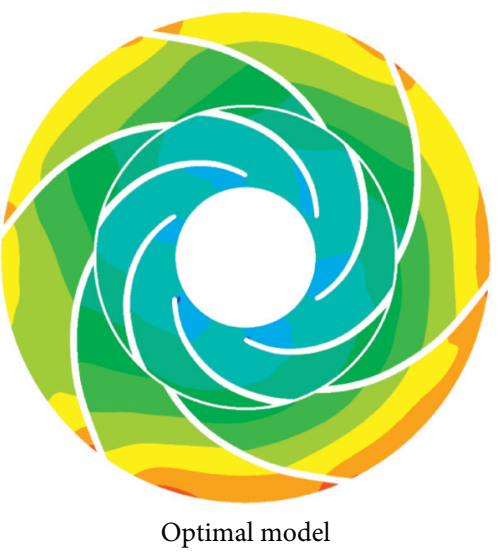

maximum pressure is achieved as the pressure increases along with the flow passage. Furthermore, the pressure distribution in the optimized model is more uniform than the original model in the blade trailing regions. The optimization effect on the pressure distribution is much significant at the design and overload flow conditions.

Figures 19-21 show the comparison of the flow path of the volute flow before and after optimization. The images were generated from the $Y Z$ plane in the volute domain, at $x=0 \mathrm{~m}$. At part loads, the streamline distribution was similar for both original and optimized models. From the volute inlet, the flow velocity reduces as the fluid moves towards the outlet region. The design point followed the same trend, except that at the outlet of the volute, the relatively low velocity region improved. At overload condition, the range of the low velocity region is significantly increased, and the low velocity region of the fluid flow is extended to the outlet of the flow channel. In the original model, an area of secondary flow appeared at the exit of the volute, and the streamline distribution was distorted. This is greatly improved after optimization with the hybrid 


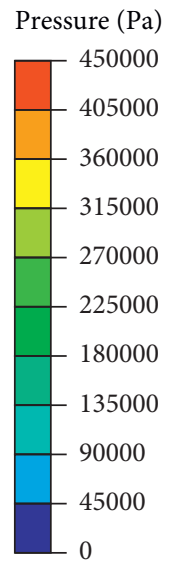

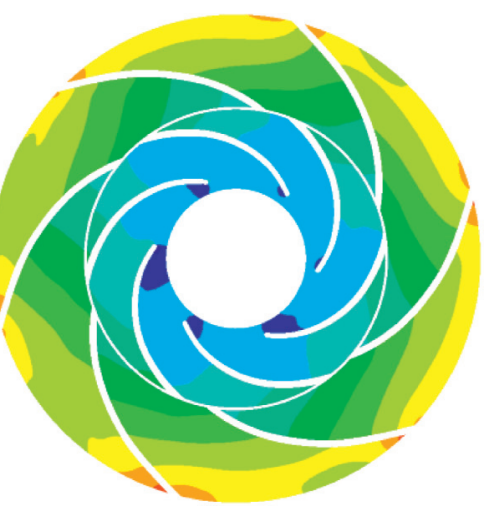

Original model

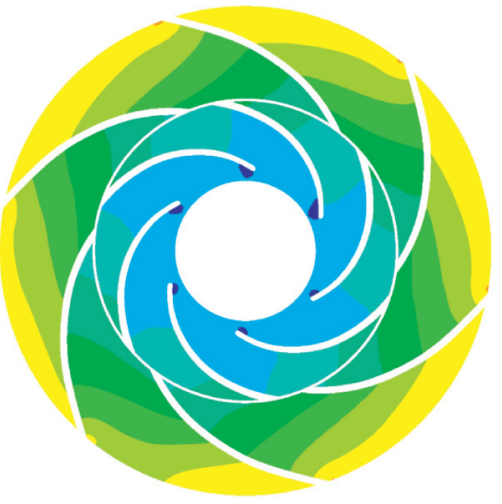

Optimal model

Figure 17: Comparison of pressure distribution $1.0 Q_{\mathrm{d}}$.

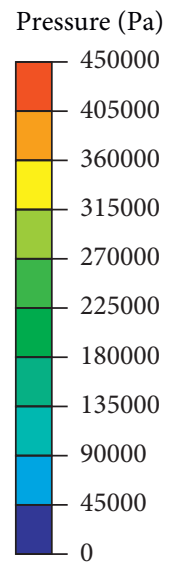

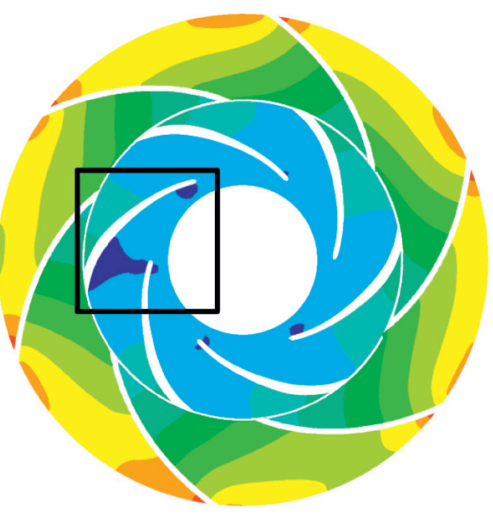

Original model

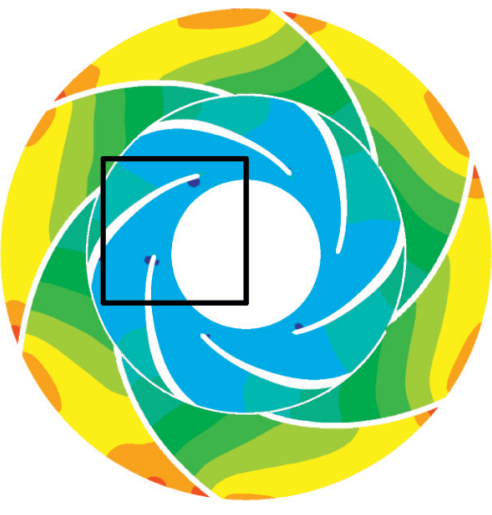

Optimal model

FIgURE 18: Comparison of pressure distribution at $1.2 Q_{\mathrm{d}}$.

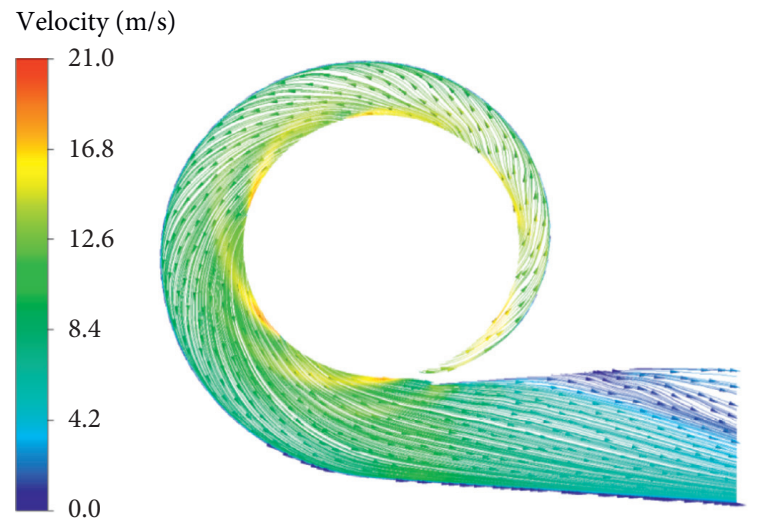

Original model

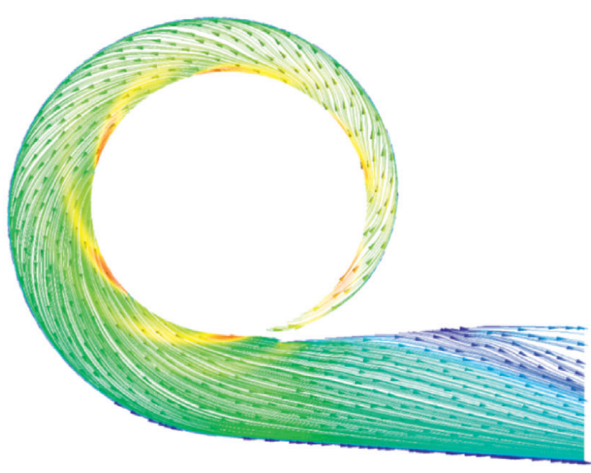

Optimal model

FIgURE 19: Velocity and streamline distribution at $0.8 Q_{\mathrm{d}}$. 


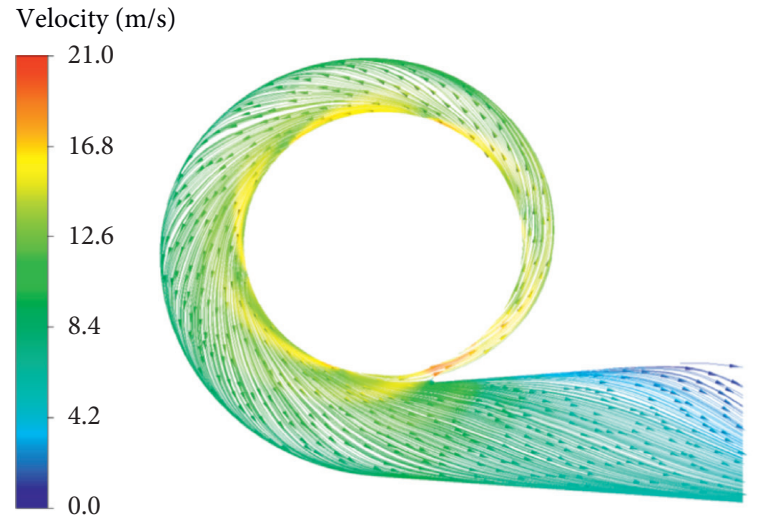

Original model

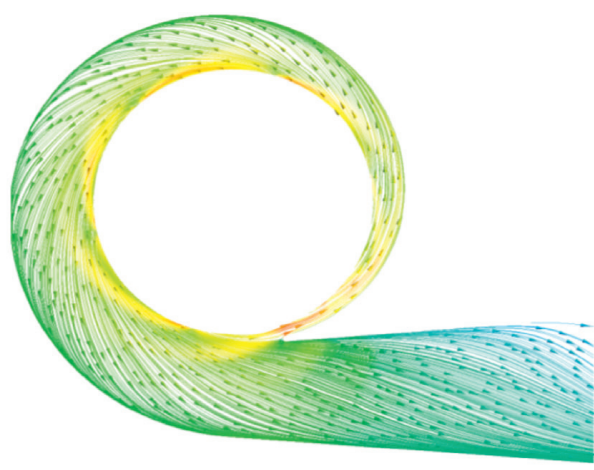

Optimal model

Figure 20: Velocity and streamline distribution at $1.0 Q_{\mathrm{d}}$.
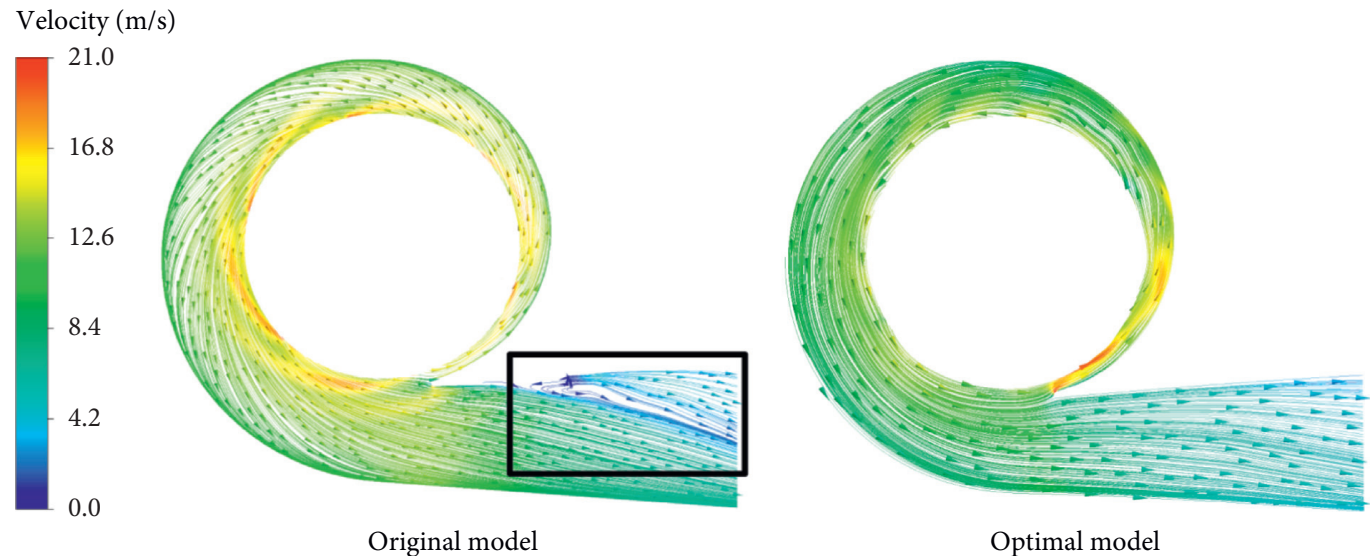

FIGURE 21: Velocity and streamline distribution at $1.2 Q_{\mathrm{d}}$.

approximate model. The flow phenomenon is greatly improved, the flow resistance is reduced, and the efficiency is increased.

\section{Conclusion}

In this paper, an optimization strategy was designed to improve the high-efficiency range of the double-suction centrifugal pump. First the "efficiency-house" theory was introduced to convert the multiple objectives into a single optimization target. The fitting principles and accuracy test methods of single approximation model and hybrid approximation model were introduced, and multiple working conditions of the double-suction pump were optimized based on ANN, Kriging, and hybrid approximation models. The following conclusions were drawn:

(1) The geometry parameters $\beta_{2 \_\mathrm{h}}, \beta_{4 \_s}, \theta_{1 \_\mathrm{h}}$, and $\theta_{2 \_s}$ had the most influence on the single optimization target based on efficiency-house

(2) The fitting accuracy of the composite approximate model is higher than that of the single approximate model, especially under the condition of a smaller sample size
(3) The accuracy of the hybrid approximation model is higher than that of the single approximation model, ensuring the reliability of the hybrid approximation model

(4) With ANN and Kriging models, a hybrid approximate model an optimization strategy was built to widen the high-efficiency range of the double-suction centrifugal pump at overload conditions by $1.63 \%, 1.95 \%$, and $4.94 \%$ under for flow conditions $0.8 Q_{\mathrm{d}}, 1.0 Q_{\mathrm{d}}$, and $1.2 Q_{\mathrm{d}}$, respectively

(5) The TKE distribution was reduced, and the secondary flow phenomena towards the exit of the volute during overload conditions improved significantly after optimization.

\section{Nomenclature}

$b_{2}$ : Blade width, $\mathrm{mm}$

$D$ : Impeller diameter, $\mathrm{mm}$

$H$ : Head, $\mathrm{m}$

$k$ : Kinetic energy of turbulence, $\mathrm{m}^{2} / \mathrm{s}^{2}$

$N$ : Rotational speed, $r / m i n$

$p$ : Pressure, $\mathrm{Pa}$ 
$P_{S}:$ Shaft power, $\mathrm{kW}$

Q: Flow rate, $\mathrm{m}^{3} / \mathrm{h}$

$S$ : Area of efficiency-house

u: Velocity, $\mathrm{m} / \mathrm{s}$

$z$ : Number of blades.

Greek Symbols

$\beta_{1 \_\mathrm{h}}$ : Blade angle at hub inlet,

$\beta_{2 \_}$: Blade angle at hub exit, ${ }^{\circ}$

$\beta_{3 \_s}$ : Blade angle at shroud inlet,

$\beta_{4 \_s}: \quad$ Blade angle at shroud exit, ${ }^{\circ}$

$\theta_{1 \_\mathrm{h}}: \quad$ Leading edge position at hub,

$\theta_{2 \_}: \quad$ Leading edge position at shroud,

$\Delta \theta_{1 \_\mathrm{h}}$ : Leading edge wrap angle at hub, ${ }^{\circ}$

$\Delta \theta_{2 \_}$: Leading edge wrap angle at shroud,

$\varepsilon: \quad$ Turbulence dissipation rate, $\mathrm{m}^{2} / \mathrm{s}^{3}$

$\eta: \quad$ Efficiency, \%

$\rho: \quad$ Density, $\mathrm{kg} / \mathrm{m}^{3}$

$\mu$ : $\quad$ Dynamic viscosity, Pa.s

$\mu_{t}$ : Turbulent viscosity, $\mathrm{m}^{2} / \mathrm{s}$

$\square: \quad$ Specific dissipation of turbulent kinetic energy, $\mathrm{s}^{-1}$

$\varphi$ : $\quad$ Flow coefficient.

Abbreviations

ANN: Artificial neural network

DOE: Design of experiment

GCI: $\quad$ Grid convergence index

LHS: Latin hypercube sampling

MOGA: Multiobjective generic algorithm

NPSH: Net positive suction head, $\mathrm{m}$

NSGA: Nondominated sorting genetic algorithm

PSO: Particle swarm optimization

RANS: Reynolds-averaged Navier-Stokes

RBNN: Radial bias neural network

SST: $\quad$ Shear stress transport.

\section{Data Availability}

The data belongs to National Research Center of Pumps, Jiangsu University, China, and therefore cannot be made freely available. Requests for access to these data should be made to the corresponding author.

\section{Conflicts of Interest}

The authors declare that there are no conflicts of interest regarding the publication of this article.

\section{Authors' Contributions}

WW and MKO conceived and designed the study and analyzed the most of results. JP and SY refined the ideas and carried out additional analyses. MKO wrote the manuscript, and JC carried out the performance measurement in the lab. FKO proofread and edited of the final draft. The final manuscript was read and approved by all authors.

\section{Acknowledgments}

This work was supported by the Natural Science Foundation of Jiangsu Province (Grant no. BK20190851), Primary
Research \& Development Plan of Shandong Province (Grant no. 2019TSLH0304), Natural Science Foundation of China (Grant no. 51879121), Primary Research \& Development Plan of Jiangsu Province (Grant no. BE2019009-1), Natural Science Foundation of the Jiangsu Higher Education Institutions of China (No. 18KJB470005), and China Postdoctoral Science Foundation (No. 2018M640462).

\section{References}

[1] W. Wang, G. Pavesi, J. Pei, and S. Yuan, "Transient simulation on closure of wicket gates in a high-head Francis-type reversible turbine operating in pump mode," Renewable Energy, vol. 145, pp. 1817-1830, 2020.

[2] J. Pei, W. Wang, M. K. Osman, and X. Gan, "Multiparameter optimization for the nonlinear performance improvement of centrifugal pumps using a multilayer neural network," Journal of Mechanical Science and Technology, vol. 33, no. 6, pp. 2681-2691, 2019.

[3] M. K. Osman, W. Wang, J. Yuan, J. Zhao, Y. Wang, and J. Liu, "Flow loss analysis of a two-stage axially split centrifugal pump with double inlet under different channel designs," Proceedings of the Institution of Mechanical Engineers, Part C: Journal of Mechanical Engineering Science, vol. 233, no. 15, pp. 5316-5328, 2019.

[4] J. Pei, W. Wang, G. Pavesi, M. K. Osman, and F. Meng, "Experimental investigation of the nonlinear pressure fluctuations in a residual heat removal pump," Annals of Nuclear Energy, vol. 131, pp. 63-79, 2019.

[5] J. Pei, M. K. Osman, W. Wang, J. Yuan, T. Yin, and D. Appiah, "Unsteady flow characteristics and cavitation prediction in the double-suction centrifugal pump using a novel approach," Proceedings of the Institution of Mechanical Engineers, Part A: Journal of Power and Energy, vol. 234, no. 3, pp. 283-299, 2020.

[6] W. Wang, M. K. Osman, J. Pei, X. Gan, and T. Yin, “Artificial neural networks approach for a multi-objective cavitation optimization design in a double-suction centrifugal pump," Processes, vol. 7, no. 5, p. 246, 2019.

[7] H. W. Oh and K.-Y. Kim, "Conceptual design optimization of mixed-flow pump impellers using mean streamline analysis," Proceedings of the Institution of Mechanical Engineers, Part A: Journal of Power and Energy, vol. 215, no. 1, pp. 133-138, 2001.

[8] H. W. Oh and M. K. Chung, "Optimum values of design variables versus specific speed for centrifugal pumps," Proceedings of the Institution of Mechanical Engineers, Part A: Journal of Power and Energy, vol. 213, no. 3, pp. 219-226, 1999.

[9] J.-H. Kim, H.-C. Lee, J.-H. Kim et al., "Improvement of hydrodynamic performance of a multiphase pump using design of experiment techniques," Journal of Fluids Engineering, vol. 137, no. 8, 2015.

[10] R. Spence and J. Amaral-Teixeira, "A CFD parametric study of geometrical variations on the pressure pulsations and performance characteristics of a centrifugal pump," Computers \& Fluids, vol. 38, no. 6, pp. 1243-1257, 2009.

[11] J. Pei, X. Gan, W. Wang, S. Yuan, and Y. Tang, "Multi-objective shape optimization on the inlet pipe of a vertical inline pump," Journal of Fluids Engineering, vol. 141, no. 6, 2019.

[12] N. Timnak and A. Jahangirian, "Multi-point optimization of transonic airfoils using an enhanced genetic algorithm," Proceedings of the Institution of Mechanical Engineers, Part G: 
Journal of Aerospace Engineering, vol. 232, no. 7, pp. 13471360, 2018.

[13] R. Jin, C. Wei, and T. W. Simpson, "Comparative studies of metamodelling techniques under multiple modelling criteria," Structural and Multidisciplinary Optimization, vol. 23, no. 1, pp. 1-13, 2001.

[14] W. Wang, J. Pei, S. Yuan, J. Zhang, J. Yuan, and C. Xu, "Application of different surrogate models on the optimization of centrifugal pump," Journal of Mechanical Science and Technology, vol. 30, no. 2, pp. 567-574, 2016.

[15] F. Meng, Y. Li, S. Yuan, W. Wang, Y. Zheng, and M. K. Osman, "Multiobjective combination optimization of an impeller and diffuser in a reversible axial-flow pump based on a two-layer artificial neural network," Processes, vol. 8, no. 3, p. 309, 2020.

[16] S. Derakhshan, M. Pourmahdavi, E. Abdolahnejad, A. Reihani, and A. Ojaghi, "Numerical shape optimization of a centrifugal pump impeller using artificial bee colony algorithm," Computers \& Fluids, vol. 81, pp. 145-151, 2013.

[17] A. Nourbakhsh, H. Safikhani, and S. Derakhshan, "The comparison of multi-objective particle swarm optimization and NSGA II algorithm: applications in centrifugal pumps," Engineering Optimization, vol. 43, no. 10, pp. 1095-1113, 2011.

[18] R. B. Medvitz, R. F. Kunz, D. A. Boger, J. W. Lindau, A. M. Yocum, and L. L. Pauley, "Performance analysis of cavitating flow in centrifugal pumps using multiphase CFD," Journal of Fluids Engineering, vol. 124, no. 2, pp. 377-383, 2002.

[19] F. R. Menter, "Two-equation eddy-viscosity turbulence models for engineering applications," AIAA Journal, vol. 32, no. 8, pp. 1598-1605, 1994.

[20] J. Bardina, P. Huang, T. Coakley, J. Bardina, P. Huang, and T. Coakley, "Turbulence modeling validation," in Proceedings of the 28th Fluid Dynamics Conference, Snowmass Village, CO, USA, June 1997.

[21] J. Pei, M. K. Osman, W. Wang, J. Yuan, T. Yin, and D. Appiah, "Unsteady flow characteristics and cavitation prediction in the double-suction centrifugal pump using a novel approach," Proceedings of the Institution of Mechanical Engineers, Part A: Journal of Power and Energy, 2019.

[22] J. Pei, F. Zhang, D. Appiah et al., "Performance prediction based on effects of wrapping angle of a side channel pump," Energies, vol. 12, no. 1, p. 139, 2019.

[23] H.-l. Liu, M.-m. Liu, Y. Bai, and L. Dong, "Effects of mesh style and grid convergence on numerical simulation accuracy of centrifugal pump," Journal of Central South University, vol. 22, no. 1, pp. 368-376, 2015.

[24] X. Tang, M. Zou, F. Wang, X. Li, and X. Shi, "Comprehensive numerical investigations of unsteady internal flows and cavitation characteristics in double-suction centrifugal pump," Mathematical Problems in Engineering, vol. 2017, 2017.

[25] J. F. Gülich, Centrifugal Pumps, Springer Science \& Business Media, Berlin, Germany, 2010.

[26] J. Pei, M. K. Osman, W. Wang, D. Appiah, T. Yin, and Q. Deng, "A practical method for speeding up the cavitation prediction in an industrial double-suction centrifugal pump," Energies, vol. 12, no. 11, p. 2088, 2019.

[27] J. Pei, W.-j. Wang, and S.-q. Yuan, "Statistical analysis of pressure fluctuations during unsteady flow for low-specificspeed centrifugal pumps," Journal of Central South University, vol. 21, no. 3, pp. 1017-1024, 2014.

[28] J. Pei, T. Yin, S. Yuan, W. Wang, and J. Wang, "Cavitation optimization for a centrifugal pump impeller by using orthogonal design of experiment," Chinese Journal of Mechanical Engineering, vol. 30, no. 1, pp. 103-109, 2017.

[29] L. Zhou, W. Shi, and S. Wu, "Performance optimization in a centrifugal pump impeller by orthogonal experiment and numerical simulation," Advances in Mechanical Engineering, vol. 5, Article ID 385809, 2013.

[30] T. W. Simpson, T. M. Mauery, J. Korte, and F. Mistree, "Kriging models for global approximation in simulationbased multidisciplinary design optimization," AIAA Journal, vol. 39, no. 12, pp. 2233-2241, 2001.

[31] Y. Zhang, S. Hu, J. Wu, Y. Zhang, and L. Chen, "Multi-objective optimization of double suction centrifugal pump using Kriging metamodels," Advances in Engineering Software, vol. 74, pp. 16-26, 2014.

[32] J. Kennedy and R. Eberhart, "Particle swarm optimization," in Proceedings of the ICNN'95-International Conference on Neural Networks, IEEE, Perth, Australia, December 1995.

[33] Y. Shi and R. Eberhart, "A modified particle swarm optimizer," in Proceedings of the 1998 IEEE International Conference on Evolutionary Computation Proceedings. IEEE World Congress on Computational Intelligence (Cat. No. 98TH8360), IEEE, Anchorage, AK, USA, May 1998.

[34] T. Goel, R. T. Haftka, W. Shyy, and N. V. Queipo, "Ensemble of surrogates," Structural and Multidisciplinary Optimization, vol. 33, no. 3, pp. 199-216, 2007.

[35] E. Acar and M. Rais-Rohani, "Ensemble of metamodels with optimized weight factors," Structural and Multidisciplinary Optimization, vol. 37, no. 3, pp. 279-294, 2009. 